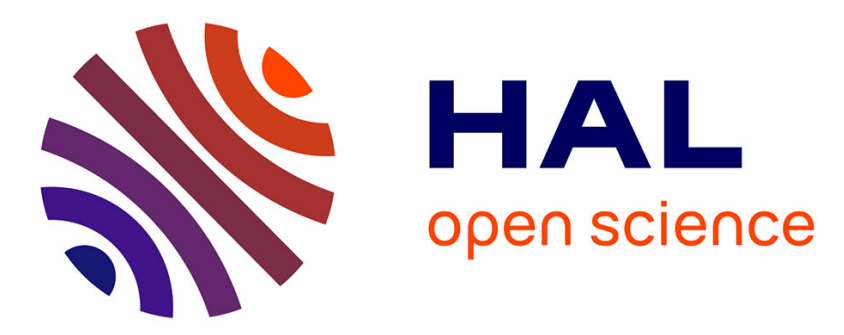

\title{
CONVERGENCE OF A FINITE VOLUME SCHEME FOR THE CONVECTION-DIFFUSION EQUATION WITH L 1 DATA
}

Thierry Gallouët, Aurélien Larcher, Jean-Claude Latché

\section{- To cite this version:}

Thierry Gallouët, Aurélien Larcher, Jean-Claude Latché. CONVERGENCE OF A FINITE VOLUME SCHEME FOR THE CONVECTION-DIFFUSION EQUATION WITH L 1 DATA. Mathematics of Computation, 2012, 81 (279), 10.1090/S0025-5718-2011-02571-8 . hal-01283569

\section{HAL Id: hal-01283569 \\ https://hal.science/hal-01283569}

Submitted on 5 Mar 2016

HAL is a multi-disciplinary open access archive for the deposit and dissemination of scientific research documents, whether they are published or not. The documents may come from teaching and research institutions in France or abroad, or from public or private research centers.
L'archive ouverte pluridisciplinaire HAL, est destinée au dépôt et à la diffusion de documents scientifiques de niveau recherche, publiés ou non, émanant des établissements d'enseignement et de recherche français ou étrangers, des laboratoires publics ou privés. 


\title{
CONVERGENCE OF A FINITE VOLUME SCHEME FOR THE CONVECTION-DIFFUSION EQUATION WITH L ${ }^{1}$ DATA
}

\author{
T. GALLOUËT, A. LARCHER, AND J.C. LATCHÉ
}

\begin{abstract}
In this paper, we prove the convergence of a finite-volume scheme for the time-dependent convection-diffusion equation with an $\mathrm{L}^{1}$ right-hand side. To this purpose, we first prove estimates for the discrete solution and for its discrete time and space derivatives. Then we show the convergence of a sequence of discrete solutions obtained with more and more refined discretizations, possibly up to the extraction of a subsequence, to a function which mets the regularity requirements of the weak formulation of the problem; to this purpose, we prove a compactness result, which may be seen as a discrete analogue to Aubin-Simon's lemma. Finally, such a limit is shown to be indeed a weak solution.
\end{abstract}

\section{INTRODUCTION}

We address in this paper the discretization by a finite volume method of the following problem:

$$
\begin{array}{ll}
\partial_{t} u+\operatorname{div}(u \boldsymbol{v})-\Delta u=f & \text { in } \Omega \times(0, T), \\
u(\boldsymbol{x}, 0)=u_{0}(\boldsymbol{x}) & \text { a.e. in } \Omega, \\
u(\boldsymbol{x}, t)=0 & \text { a.e. in } \partial \Omega \times(0, T),
\end{array}
$$

where $\Omega$ is an open, bounded, connected subset of $\mathbb{R}^{d}, d=2$ or $d=3$, which is supposed to be polygonal $(d=2)$ or polyhedral $(d=3)$, and $\partial \Omega$ stands for its boundary. The right-hand side, $f$, and the initial condition, $u_{0}$, are supposed to satisfy:

$$
f \in \mathrm{L}^{1}(\Omega \times(0, T)), \quad u_{0} \in \mathrm{L}^{1}(\Omega) .
$$

The vector-valued velocity field $\boldsymbol{v}$ is supposed to be divergence-free, to vanish on the boundary of the domain and to be regular, let us say:

$$
\begin{aligned}
& \boldsymbol{v} \in \mathrm{C}^{1}(\bar{\Omega} \times[0, T]), \\
& \operatorname{div} \boldsymbol{v}(\boldsymbol{x}, t)=0, \forall(\boldsymbol{x}, t) \in \Omega \times(0, T), \\
& \boldsymbol{v}(\boldsymbol{x}, t)=0, \forall(\boldsymbol{x}, t) \in \partial \Omega \times(0, T) .
\end{aligned}
$$

Definition 1.1 (Weak solution). We define a weak solution $u$ to problem (1.1)(1.3) by:

$$
u \in \cup_{1 \leq q<(d+2) /(d+1)} \mathrm{L}^{q}\left(0, T ; \mathrm{W}_{0}^{1, q}(\Omega)\right)
$$

2000 Mathematics Subject Classification.

Key words and phrases. Convection-diffusion equation, finite volumes, irregular data. 
and, $\forall \varphi \in \mathrm{C}_{c}^{\infty}(\Omega \times[0, T))$ :

$$
\begin{aligned}
-\int_{\Omega \times(0, T)} u(\boldsymbol{x}, t) \partial_{t} \varphi(\boldsymbol{x}, t) \mathrm{d} \boldsymbol{x} \mathrm{d} t-\int_{\Omega \times(0, T)} u(\boldsymbol{x}, t) \boldsymbol{v}(\boldsymbol{x}, t) \cdot \nabla \varphi(\boldsymbol{x}, t) \mathrm{d} \boldsymbol{x} \mathrm{d} t \\
+\int_{\Omega \times(0, T)} \nabla u(\boldsymbol{x}, t) \cdot \nabla \varphi(\boldsymbol{x}, t) \mathrm{d} \boldsymbol{x} \mathrm{d} t \\
\quad=\int_{\Omega \times(0, T)} f(\boldsymbol{x}, t) \varphi(\boldsymbol{x}, t) \mathrm{d} \boldsymbol{x} \mathrm{d} t+\int_{\Omega} u_{0}(\boldsymbol{x}) \varphi(\boldsymbol{x}, 0) \mathrm{d} \boldsymbol{x} .
\end{aligned}
$$

The existence of such a weak solution (and to more general nonlinear elliptic and parabolic problems) has been proven in [4]; further developments (in particular, concerning the uniqueness of solutions) can be found in $[1,2,3,20,11]$.

The motivation of this study lies in the fact that Problem (1.1)-(1.3) is a model problem for a class of convection-diffusion-reaction equations with $\mathrm{L}^{1}$-data encountered in the so-called Reynolds Averaged Navier-Stokes (RANS) modeling of turbulent flows. In this class of models, the effects of turbulent stresses are taken into account by an additional diffusion term in the momentum balance equation of the averaged Navier-Stokes system governing the evolution of the mean velocity field $\overline{\boldsymbol{v}}$ and pressure $\bar{p}$. This system of equations is given here for reference, in the case of incompressible flows, with $\mu$ the laminar viscosity and $\mathbf{g}$ a forcing-term:

$$
\begin{aligned}
& \partial_{t} \overline{\boldsymbol{v}}+\operatorname{div}(\overline{\boldsymbol{v}} \otimes \overline{\boldsymbol{v}})-\operatorname{div}\left(\left(\mu+\mu_{t}(\cdot)\right)\left(\nabla \overline{\boldsymbol{v}}+\nabla \overline{\boldsymbol{v}}^{t}\right)\right)+\nabla \bar{p}=\mathbf{g}, \\
& \operatorname{div} \overline{\boldsymbol{v}}=0 .
\end{aligned}
$$

The additional diffusivity $\mu_{t}$, called "turbulent viscosity", needs to be modelled by an algebraic relation. Usually, this relation involves a set of characteristic turbulent scales $\left(\chi_{i}\right)_{0<i<n}$; for instance the turbulent kinetic energy $k\left(\mathrm{~m}^{2} / \mathrm{s}^{2}\right)$, its dissipation rate $\varepsilon\left(\mathrm{m}^{2} / \mathrm{s}^{3}\right)$ or the turbulent frequency scale $\omega\left(\mathrm{s}^{-1}\right)$ are used in two-equation models like the $k-\varepsilon$ model of Launder-Spalding and the $k-\omega$ model of Wilcox. Turbulent scales themselves have to be computed by solving a set of scalar convection-diffusion equations, commonly called "turbulent transport equations", which share the same structure:

$$
\partial_{t} \chi_{i}+\operatorname{div}\left(\chi_{i} \overline{\boldsymbol{v}}\right)-\operatorname{div}\left(\lambda\left(\left\{\chi_{p}\right\}_{0<p \leq n}\right) \nabla \chi_{i}\right)=f_{\chi_{i}}\left(\left\{\chi_{p}\right\}_{0<p \leq n}\right) .
$$

In these equations, following from the Boussinesq hypothesis, every source term $f_{\chi_{i}}$ is linear (with a bounded coefficient) with respect to $|\nabla \overline{\boldsymbol{v}}|^{2}=\sum_{i, j=1}^{d}\left(\partial_{j} \bar{v}_{i}\right)^{2}$, with $\bar{v}_{i}$ the $i$-th component of $\overline{\boldsymbol{v}}$. Since $\overline{\boldsymbol{v}}$ satisfies the classical energy estimate of the Navier-Stokes analysis, which can be derived from System (1.4), $\nabla \overline{\boldsymbol{v}}$ belongs to $\mathrm{L}^{2}(\Omega \times(0, T))$, and the right-hand side of Equation (1.5) lies in $\mathrm{L}^{1}(\Omega \times(0, T))$.

Let us mention that convection-diffusion equations with $\mathrm{L}^{1}$ data are also encountered in electrodynamics modeling [6] or heating by induction [8].

In this paper, we show that a sequence of approximate solutions obtained by a backward-in-time and upwind finite volume method converges up to a subsequence towards a function $\bar{u}$ which is a weak solution of the problem, in the sense of Definition 1.1. To this purpose, we extend to the time-dependent case techniques developed for steady problems with $\mathrm{L}^{1}$ data, for a single elliptic equation in $[16,10]$ and for a system of two coupled elliptic equations arising in heat dissipation by the 
Joule effect in [6]. Note that all of these works (including the present one) only apply to the standard two-point approximation of the diffusion flux (and so to particuliar meshes, see remark 2.1), the extension to more general finite volume schemes being still an open problem. Similar studies in the finite element framework, for a single elliptic equation and with meshes still satisfying similar assumptions, may be found in $[17,7]$.

The presentation is organized as follows. In Section 2, we define the approximation spaces and describe the discrete functional analysis framework which is used in the subsequent developments. Then we prove an abstract compactness result, which may be considered as a discrete analogue of the classical Aubin-Simon's lemma (Section 3). The scheme is then given (Section 4), then we derive estimates satisfied by the discrete solution (Section 5 ), and conclude by the convergence analysis (Section 6).

\section{Discrete spaces and Functional Framework}

An admissible finite volume discretization of $\Omega$, denoted by $\mathcal{D}$, is given by $\mathcal{D}=$ $(\mathcal{M}, \mathcal{E}, \mathcal{P})$, where:

(1) $\mathcal{M}$ is a finite family of non empty open polygonal $(d=2)$ or polyhedral $(d=3)$ convex disjoint subsets of $\Omega$ (the "control volumes") such that $\bar{\Omega}=\cup_{K \in \mathcal{M}} \bar{K}$. For any $K \in \mathcal{M}$, let $\partial K=\bar{K} \backslash K$ be the boundary of $K$.

(2) $\mathcal{E}$ is a finite family of disjoint subsets of $\bar{\Omega}$ (the "faces" of the mesh), such that, for all $\sigma \in \mathcal{E}$, there exists a hyperplane $E$ of $\mathbb{R}^{d}$ and $K \in \mathcal{M}$ with $\bar{\sigma}=\partial K \cap E$ and $\sigma$ is a non empty open subset of $E$. We assume that, for all $K \in \mathcal{M}$, there exists a subset $\mathcal{E}(K)$ of $\mathcal{E}$ such that $\partial K=\cup_{\sigma \in \mathcal{E}(K)} \bar{\sigma}$. For all $\sigma \in \mathcal{E}$, we suppose that either $\sigma \subset \partial \Omega$ or there exists $(K, L) \in \mathcal{M}^{2}$ with $K \neq L$ such that $\bar{K} \cap \bar{L}=\bar{\sigma}$; we denote in the latter case $\sigma=K \mid L$. We denote by $\mathcal{E}_{\text {ext }}$ the set of the faces included in $\partial \Omega$ and $\mathcal{E}_{\text {int }}=\mathcal{E} \backslash \mathcal{E}_{\text {ext }}$ the set of internal faces.

(3) $\mathcal{P}$ is a family of points of $\Omega$ indexed by $\mathcal{M}$, denoted by $\mathcal{P}=\left(\boldsymbol{x}_{K}\right)_{K \in \mathcal{M}}$. The family $\mathcal{P}$ is supposed to be such that, for all $K \in \mathcal{M}, \boldsymbol{x}_{K} \in K$. Furthermore, for all $\sigma \in \mathcal{E}_{\text {int }}, \sigma=K \mid L$, it is assumed that the straight line $\left(\boldsymbol{x}_{K}, \boldsymbol{x}_{L}\right)$ going through $\boldsymbol{x}_{K}$ and $\boldsymbol{x}_{L}$ is orthogonal to $K \mid L$.

Remark 2.1. The possibility to build the family $\mathcal{P}$ restricts in practice the choice for the mesh. In applications, we only use rectangles (or rectangular hexahedra in $3 D)$, acute angle simplices or Voronoï cells.

By $|K|$ and $|\sigma|$, we denote hereafter respectively the measure of the control volume $K$ and of the face $\sigma$. For any control volume $K$ and face $\sigma$ of $K$, we denote by $d_{K, \sigma}$ the Euclidean distance between $\boldsymbol{x}_{K}$ and $\sigma$ and by $\boldsymbol{n}_{K, \sigma}$ the unit vector normal to $\sigma$ outward from $K$. For any face $\sigma$, we define $d_{\sigma}=d_{K, \sigma}+d_{L, \sigma}$, if $\sigma$ separates the two control volumes $K$ and $L$ (in which case $d_{\sigma}$ is the Euclidean distance between $\boldsymbol{x}_{K}$ and $\boldsymbol{x}_{L}$ ) and $d_{\sigma}=d_{K, \sigma}$ if $\sigma$ is included in the boundary. For any control volume $K \in \mathcal{M}, h_{K}$ stands for the diameter of $K$. We denote by $h_{\mathcal{M}}$ the quantity $h_{\mathcal{M}}=\max _{K \in \mathcal{M}} h_{K}$. 
Let $\mathrm{H}_{\mathcal{M}} \subset \mathrm{L}^{\infty}(\Omega)$ be the space of functions piecewise constant over any cell $K \in \mathcal{M}$. For a finite $q \geq 1$, we define a discrete $\mathrm{W}_{0}^{1, q}$-norm by:

$$
\|u\|_{1, q, \mathcal{M}}^{q}=\sum_{\sigma \in \mathcal{E}_{\mathrm{int}}, \sigma=K \mid L}|\sigma| d_{\sigma}\left|\frac{u_{K}-u_{L}}{d_{\sigma}}\right|^{q}+\sum_{\sigma \in \mathcal{E}_{\mathrm{ext}}, \sigma \in \mathcal{E}(K)}|\sigma| d_{\sigma}\left|\frac{u_{K}}{d_{\sigma}}\right|^{q} .
$$

We also define:

$$
\begin{aligned}
\|u\|_{1, \infty, \mathcal{M}}= & \max \left\{\left\{\frac{\left|u_{K}-u_{L}\right|}{d_{\sigma}}, \sigma \in \mathcal{E}_{\text {int }}, \sigma=K \mid L\right\}\right. \\
& \left.\cup\left\{\frac{\left|u_{K}\right|}{d_{\sigma}}, \sigma \in \mathcal{E}_{\text {ext }}, \sigma \in \mathcal{E}(K)\right\} \cup\left\{\left|u_{K}\right|, K \in \mathcal{M}\right\}\right\}
\end{aligned}
$$

For $q>1$, we associate to this norm a dual norm with respect to the $\mathrm{L}^{2}$ inner product, denoted by $\|\cdot\|_{-1, q^{\prime}, \mathcal{M}}$ with $q^{\prime}$ given by $1 / q+1 / q^{\prime}=1$ if $q$ is finite and $q^{\prime}=1$ if $q=+\infty$, and defined by:

$$
\|u\|_{-1, q^{\prime}, \mathcal{M}}=\sup _{v \in \mathrm{H}_{\mathcal{M}}(\Omega), v \neq 0} \frac{1}{\|v\|_{1, q, \mathcal{M}}} \int_{\Omega} u v \mathrm{~d} \boldsymbol{x} .
$$

As a consequence of the discrete Hölder inequality, the following bound holds for any $q, r \in[1,+\infty]$ such that $q \leq r$ :

$$
\forall u \in \mathrm{H}_{\mathcal{M}}, \quad\|u\|_{1, q, \mathcal{M}} \leq(d|\Omega|)^{1 / q-1 / r}\|u\|_{1, r, \mathcal{M}}
$$

and, consequently, for any $q, r \in[1,+\infty]$ such that $q \leq r$ :

$$
\forall u \in \mathrm{H}_{\mathcal{M}}, \quad\|u\|_{-1, q, \mathcal{M}} \leq(d|\Omega|)^{1 / q-1 / r}\|u\|_{-1, r, \mathcal{M}} .
$$

We denote by $\xi_{\mathcal{M}}>0$ a positive real number such that:

$$
\forall K \in \mathcal{M}, \forall \sigma \in \mathcal{E}(K), \quad \xi_{\mathcal{M}} \leq \frac{d_{K, \sigma}}{d_{\sigma}}, \quad \text { and } \quad \xi_{\mathcal{M}} \leq \frac{d_{K, \sigma}}{h_{K}} .
$$

The greatest real number satisfying these inequalities may be considered as a measure of the regularity of the mesh.

The following discrete Sobolev inequalities are proven in [12, Lemma 9.5, p.790] and $[9,13]$.

Lemma 2.2 (Discrete Sobolev inequality). For any $q \in[1, d)$, there exists a real number $C\left(\Omega, \xi_{\mathcal{M}}, q\right)>0$ such that:

$$
\|u\|_{L^{q^{\star}}(\Omega)} \leq C\left(\Omega, \xi_{\mathcal{M}}, q\right)\|u\|_{1, q, \mathcal{M}} \quad \text { with } \quad q^{\star}=\frac{d q}{d-q} .
$$

For $q \geq d$ and any $p \in[1,+\infty)$, there exists a real number $C\left(\Omega, \xi_{\mathcal{M}}, p\right)>0$ such that:

$$
\|u\|_{\mathrm{L}^{p}(\Omega)} \leq C\left(\Omega, \xi_{\mathcal{M}}, p\right)\|u\|_{1, q, \mathcal{M}}
$$

In addition, the following bound is proven in [13, Lemma 5.4].

Lemma 2.3 (Space translates estimates). Let $v \in \mathrm{H}_{\mathcal{M}}$, and let $\bar{v}$ be its extension by 0 to $\mathbb{R}^{d}$. Then:

$$
\|\bar{v}(\cdot+\boldsymbol{y})-\bar{v}(\cdot)\|_{\mathrm{L}^{1}\left(\mathbb{R}^{d}\right)} \leq \sqrt{d}|\boldsymbol{y}|\|v\|_{1,1, \mathcal{M}}, \quad \forall \boldsymbol{y} \in \mathbb{R}^{d} .
$$


The following result is a consequence of the Kolmogorov's theorem and of this inequality.

Theorem 2.1. Let $\mathcal{M}^{k}$ be a sequence of meshes the step of which tends to zero, and regular in the sense that any $\mathcal{M}^{k}, k \in \mathbb{N}$, satisfies the regularity assumption (2.3) with a unique (i.e. independent of $k$ ) positive real number $\xi$.

Let $q \in[1,+\infty)$, and let $\left(u_{\mathcal{M}}^{k}\right)_{k \in \mathbb{N}}$ be a sequence of discrete functions (i.e. such that, $\forall k \in \mathbb{N}, u^{k} \in \mathrm{H}_{\mathcal{M}}^{k}$, where $\mathrm{H}_{\mathcal{M}}^{k}$ is the discrete space associated to $\mathcal{M}^{k}$ ) satisfying:

$$
\forall k \in \mathbb{N}, \quad\left\|u^{k}\right\|_{1, q, \mathcal{M}} \leq C
$$

where $C$ is a given positive real number. Then, possibly up to the extraction of a subsequence, the sequence $\left(u_{\mathcal{M}}^{k}\right)_{k \in \mathbb{N}}$ converges in $\mathrm{L}^{p}(\Omega)$ to a function $u \in \mathrm{W}_{0}^{1, q}(\Omega)$, for any $p \in\left[1, q^{\star}\right)$, where $q^{\star}=d q /(d-q)$ if $q<d$ and $q^{\star}=+\infty$ otherwise.

Remark 2.4 (Extension of Theorem 2.1 to $q=+\infty$ ). If we now suppose that, with the same notations:

$$
\forall k \in \mathbb{N}, \quad\left\|u^{k}\right\|_{1, \infty, \mathcal{M}} \leq C,
$$

then, still up to the extraction of a subsequence, $\left(u_{\mathcal{M}}^{k}\right)_{k \in \mathbb{N}}$ may be shown to converge in any $\mathrm{L}^{p}(\Omega)$ with $p \in[1,+\infty)$ to a limit $u$ which belongs to $\mathrm{W}_{0}^{1, \infty}(\Omega)$, the regularity of $u$ being a consequence of the fact that its gradient is bounded in any $\mathrm{L}^{p}(\Omega)^{d}$, $p \in[1,+\infty)$, by a constant which does not depend on $p$.

Furthermore, we suppose given a uniform partition of the time-interval $[0, T)$, such that $[0, T]=\cup_{0 \leq n<N}\left[t^{n}, t^{n+1}\right]$ (so $t^{n}=n \delta t$, with $\left.\delta t=T / N\right)$. Let $\mathrm{H}_{\mathcal{D}}$ be the space of piecewise constant functions over each $K \times I^{n}$, for $K \in \mathcal{M}$ and $I^{n}=$ $\left(t^{n}, t^{n+1}\right), 0 \leq n<N$. To each sequence $\left(u^{n}\right)_{n=0, N}$ of functions of $\mathrm{H}_{\mathcal{M}}(\Omega)$, we associate the function $u \in \mathrm{H}_{\mathcal{D}}$ defined by:

$$
u(\boldsymbol{x}, t)=u^{n+1}(\boldsymbol{x}) \quad \text { for a.e. } \boldsymbol{x} \in \Omega \text {, for any } t \in\left(t^{n}, t^{n+1}\right), 0 \leq n<N .
$$

In addition, for any $u \in \mathrm{H}_{\mathcal{D}}$ and $1 \leq n \leq N$, we define $\partial_{t, \mathcal{D}}(u)^{n} \in \mathrm{H}_{\mathcal{M}}$ (referred to hereafter as the discrete time derivative of $u$ ) by:

$$
\partial_{t, \mathcal{D}}(u)^{n}(\boldsymbol{x})=\frac{u^{n}(\boldsymbol{x})-u^{n-1}(\boldsymbol{x})}{\delta t} \quad\left(\text { i.e. } \partial_{t, \mathcal{D}}(u)_{K}^{n}=\frac{u_{K}^{n}-u_{K}^{n-1}}{\delta t}, \forall K \in \mathcal{M}\right) .
$$

\section{A compactness Result}

The aim of this section is to establish a compactness result for sequences of functions of $\mathrm{H}_{\mathcal{D}}$ which are controlled in the discrete $\mathrm{L}^{1}\left(0, T ; \mathrm{W}_{0}^{1, q}(\Omega)\right)$ norm, and the discrete time derivative of which is controlled in the discrete $\mathrm{L}^{1}\left(0, T ; \mathrm{W}^{-1, r}(\Omega)\right)$ norm, $r$ not being necessarily equal to $q^{\prime}$. A new difficulty (with respect with previous analyses which can be found in [12, chapter IV] or [14]) lies in the fact that the space norms for the function and its time-derivative are not dual with respect to the $\mathrm{L}^{2}$ inner product, which leads us to derive a discrete equivalent of the Lions' lemma 3.1 below. Then we prove a discrete analogue of the Aubin-Simon compactness lemma, using the Kolmogorov theorem. 
3.1. A discrete Lion's lemma. Let us first recall the Lions' lemma [19], [5, Lemma II.5.15, p.97].

Lemma 3.1. Let $B_{0}, B_{1}, B_{2}$ be three Banach spaces such that $B_{0} \subset B_{1} \subset B_{2}$, with a compact imbedding of $B_{0}$ in $B_{1}$ and a continuous imbedding of $B_{1}$ in $B_{2}$. Then for all $\varepsilon>0$, there exists $C(\varepsilon)>0$ such that:

$$
\forall u \in B_{0}, \quad\|u\|_{B_{1}} \leq \varepsilon\|u\|_{B_{0}}+C(\varepsilon)\|u\|_{B_{2}} .
$$

Let us state a discrete version of this lemma suitable for our purpose, taking $B_{1}=\mathrm{L}^{q}(\Omega)$ and replacing $B_{0}$ and $B_{2}$ by a sequence of discrete spaces, associated to meshes the step of which tends to zero, endowed with the $\|\cdot\|_{1, q, \mathcal{M}}$ norm and the $\|\cdot\|_{-1, r, \mathcal{M}}$ norm respectively.

Lemma 3.2 (Lions lemma - Discrete $L^{p}$ version). Let $\left(\mathcal{M}^{k}\right)_{k \in \mathbb{N}}$ be a sequence of meshes the step of which tends to zero, and regular in the sense that any $\mathcal{M}^{k}$, $k \in \mathbb{N}$, satisfies the regularity assumption (2.3) with a unique (i.e. independent of k) positive real number $\xi$.

Let $q, r \in[1,+\infty)$. Then, for all $\varepsilon>0$, there exists $C(\varepsilon)>0$ only depending on $\varepsilon$, $q, r$ and $\xi$ such that, for any sequence of discrete functions $\left(u^{k}\right)_{k \in \mathbb{N}}$ (i.e. such that, $\forall k \in \mathbb{N}, u^{k} \in \mathrm{H}_{\mathcal{M}}^{k}$, where $\mathrm{H}_{\mathcal{M}}^{k}$ is the discrete space associated to $\left.\mathcal{M}^{k}\right)$ :

$$
\forall k \in \mathbb{N}, \quad\left\|u^{k}\right\|_{L^{q}} \leq \varepsilon\left\|u^{k}\right\|_{1, q, \mathcal{M}}+C(\varepsilon)\left\|u^{k}\right\|_{-1, r, \mathcal{M}} .
$$

Proof. Let us suppose that this result is wrong. Then there exists $\varepsilon>0$ and a sequence of discrete functions $\left(u^{k}\right)_{k \in \mathbb{N}}$ such that:

$$
\forall k \in \mathbb{N}, \quad\left\|u^{k}\right\|_{\mathrm{L}^{q}(\Omega)}>\varepsilon\left\|u^{k}\right\|_{1, q, \mathcal{M}}+k\left\|u^{k}\right\|_{-1, r, \mathcal{M}} .
$$

Let $\left(v^{k}\right)_{k \in \mathbb{N}}$ be given by:

$$
\forall k \in \mathbb{N}, \quad v_{k}=\frac{1}{\left\|u^{k}\right\|_{\mathrm{L}^{q}(\Omega)}} u^{k},
$$

so that, $\forall k \in \mathbb{N},\left\|v^{k}\right\|_{L^{q}(\Omega)}=1$. By (3.2), we obtain that:

$$
\forall k \in \mathbb{N}, \quad\left\|v^{k}\right\|_{L^{q}(\Omega)}>\varepsilon\left\|v^{k}\right\|_{1, q, \mathcal{M}}+k\left\|v^{k}\right\|_{-1, r, \mathcal{M}},
$$

and hence:

$$
\forall k \in \mathbb{N}, \quad\left\|v^{k}\right\|_{1, q, \mathcal{M}}<\frac{1}{\varepsilon} .
$$

Thus, thanks to Theorem 2.1, possibly up to the extraction of a subsequence, $\left(v^{k}\right)_{k \in \mathbb{N}}$ converges in $\mathrm{L}^{q}(\Omega)$ when $k \rightarrow \infty$ to a limit $v \in W_{0}^{1, q}(\Omega)$. On one side, this limit satisfies $\|v\|_{L^{q}(\Omega)}=1$. On the other side, we have, for $k \in \mathbb{N},\left\|v^{k}\right\|_{-1, r, \mathcal{M}}<$ $1 / k$. Let $\varphi \in \mathrm{C}_{c}^{\infty}(\Omega)$. For $k \in \mathbb{N}$, we denote by $\pi^{k} \varphi$ the discrete function of $\mathrm{H}_{\mathcal{M}}^{k}$ defined by $\left(\pi^{k} \varphi\right)_{K}=\varphi\left(\boldsymbol{x}_{K}\right), \forall K \in \mathcal{M}^{k}$. By the definition of the $\|\cdot\|_{1, r^{\prime}, \mathcal{M}}$ norm, we have:

$$
\left\|\pi^{k} \varphi\right\|_{1, r^{\prime}, \mathcal{M}} \leq\|\varphi\|_{\mathrm{W}^{1, \infty}(\Omega)}(d|\Omega|)^{1-1 / r} .
$$

We thus get, for $k \in \mathbb{N}$ :

$$
\left|\int v^{k} \pi^{k} \varphi \mathrm{d} \boldsymbol{x}\right| \leq\left\|v^{k}\right\|_{-1, r, \mathcal{M}}\left\|\pi^{k} \varphi\right\|_{1, r^{\prime}, \mathcal{M}}<\|\varphi\|_{\mathrm{W}^{1, \infty}(\Omega)}(d|\Omega|)^{1 / r^{\prime}} \frac{1}{k},
$$


and, passing to the limit when $k \rightarrow \infty$, since $v^{k}$ converges to $v$ in $\mathrm{L}^{q}(\Omega)$ and $\pi^{k} \varphi$ converges to $\varphi$ in $\mathrm{L}^{\infty}(\Omega)$ :

$$
\int v \varphi \mathrm{d} \boldsymbol{x}=0
$$

Since this latter relation is valid for any $\varphi \in \mathrm{C}_{c}^{\infty}(\Omega)$, this is in contradiction with the fact that $\|v\|_{L^{q}(\Omega)}=1$.

Remark 3.3. At first glance, Lemma 3.2 may seem to be slightly more general that its continuous counterpart, since it does not require an assumption on the values of $q$ and $r$ which would ensure that $\mathrm{L}^{q}(\Omega)$ is imbedded in $\mathrm{W}^{-1, r}(\Omega)$. We show in appendix $A$ that the situation is in fact the same at the continuous level, i.e. that a continuous counterpart of Inequality (3.1) also holds in the continuous case, for any $q$ and $r$ in $[1,+\infty)$.

3.2. Estimation of time translates of discrete functions. Let us first introduce some notations. For $n \in \mathbb{Z}$, we denote by $t^{n}$ the time $t^{n}=n \delta t$ (so the definition of $t^{n}$ extends to $n \in \mathbb{Z}$ the definition already given in Section 2 for $0 \leq n \leq N)$. Let $\overline{\mathrm{H}}_{\mathcal{D}}$ be the space of discrete functions defined over $\mathbb{R}^{d} \times \mathbb{R}$ by simply supposing:

(i) that the time discretization covers $\mathbb{R}$, such that $\bar{u} \in \overline{\mathrm{H}}_{\mathcal{D}}$ reads $\bar{u}=\left(\bar{u}^{n}\right)_{n \in \mathbb{Z}}$,

(ii) and that, for $n \in \mathbb{Z}, \bar{u}^{n}$ results from the extension by zero to $\mathbb{R}^{d}$ of a function of $\mathrm{H}_{\mathcal{M}}$, which we denote by $u^{n}$.

For a positive real number $\tau$, let $\chi_{\tau}^{n}: \mathbb{R} \rightarrow \mathbb{R}$ be the function defined by $\chi_{\tau}^{n}(t)=1$ if $t<t^{n}<t+\tau$ and $\chi_{\tau}^{n}(t)=0$ otherwise. Then, for a.e. $\boldsymbol{x} \in \mathbb{R}^{d}$ and a.e. $t \in \mathbb{R}$, the difference $\bar{u}(\boldsymbol{x}, t+\tau)-\bar{u}(\boldsymbol{x}, t)$ can be expanded as follows:

$$
\bar{u}(\boldsymbol{x}, t+\tau)-\bar{u}(\boldsymbol{x}, t)=\sum_{n \in \mathbb{Z}} \chi_{\tau}^{n}(t)\left(u^{n+1}(\boldsymbol{x})-u^{n}(\boldsymbol{x})\right) .
$$

In addition, the function $\chi_{\tau}^{n}$ is the characteristic function of the interval $\left(t^{n}-\tau, t^{n}\right)$ and thus:

$$
\forall n \in \mathbb{Z}, \quad \int_{\mathbb{R}} \chi_{\tau}^{n}(t) \mathrm{d} t=\tau .
$$

Lemma 3.4 (Estimate of the time translates of a function of $\overline{\mathrm{H}}_{\mathcal{D}}$ ). Let $\bar{u}=$ $\left(\bar{u}^{n}\right)_{0 \leq n<N}$ be a function of $\overline{\mathrm{H}}_{\mathcal{D}}$, let $q \in[1,+\infty)$ and $r \in[1,+\infty)$, and let us suppose that there exists a positive real number $C$ such that:

$$
\sum_{n \in \mathbb{Z}} \delta t\left\|u^{n}\right\|_{1, q, \mathcal{M}} \leq C, \quad \sum_{n \in \mathbb{Z}} \delta t\left\|\partial_{t, \mathcal{D}}(u)^{n}\right\|_{-1, r, \mathcal{M}} \leq C .
$$

Let $\epsilon>0$. Let $\delta$ be given by:

$$
\delta=\frac{\epsilon}{4|\Omega|^{1-1 / q} C} .
$$

Let $C(\delta)$ be such that for any $v \in \mathrm{H}_{\mathcal{M}}$, an inequality of the form of Lemma 3.2 holds:

$$
\|v\|_{\mathrm{L}^{q}(\Omega)} \leq \delta\|v\|_{1, q, \mathcal{M}}+C(\delta)\|v\|_{-1, r, \mathcal{M}}
$$

and, finally, let $\tau_{0}$ be given by:

$$
\tau_{0}=\frac{\epsilon}{2|\Omega|^{1-1 / q} C(\delta) C} .
$$


Then:

$$
\forall \tau \text { such that }|\tau| \leq \tau_{0}, \quad\|\bar{u}(\cdot, t+\tau)-\bar{u}(\cdot, t)\|_{\mathrm{L}^{1}\left(\mathbb{R}^{d} \times \mathbb{R}\right)} \leq \epsilon .
$$

Proof. Let $\alpha>0$ and $C(\alpha)$ be a positive real number such that, for any $v \in \mathrm{H}_{\mathcal{M}}$, an inequality of the form of Lemma 3.2 holds:

$$
\|v\|_{\mathrm{L}^{q}(\Omega)} \leq \alpha\|v\|_{1, q, \mathcal{M}}+C(\alpha)\|v\|_{-1, r, \mathcal{M}} .
$$

Let $\tau$ be a positive real number, and $\bar{u}$ be a function of $\overline{\mathrm{H}}_{\mathcal{D}}$, its restriction to $\Omega$ being denoted by $u$. We have, for $t \in \mathbb{R}$ :

$$
\begin{aligned}
& \|\bar{u}(\cdot, t+\tau)-\bar{u}(\cdot, t)\|_{\mathrm{L}^{1}\left(\mathbb{R}^{d}\right)}=\|u(\cdot, t+\tau)-u(\cdot, t)\|_{\mathrm{L}^{1}(\Omega)} \\
& \quad \leq|\Omega|^{1-1 / q}\|u(\cdot, t+\tau)-u(\cdot, t)\|_{\mathrm{L}^{q}(\Omega)} \\
& \quad \leq|\Omega|^{1-1 / q}\left[\alpha\|u(\cdot, t+\tau)-u(\cdot, t)\|_{1, q, \mathcal{M}}+C(\alpha)\|u(\cdot, t+\tau)-u(\cdot, t)\|_{-1, r, \mathcal{M}}\right] .
\end{aligned}
$$

This inequality yields:

$$
\int_{t \in \mathbb{R}}\|\bar{u}(\cdot, t+\tau)-\bar{u}(\cdot, t)\|_{\mathrm{L}^{1}\left(\mathbb{R}^{d}\right)} \mathrm{d} t \leq|\Omega|^{1-1 / q}\left[\alpha T_{1}+C(\alpha) T_{2}\right],
$$

with:

$T_{1}=\int_{t \in \mathbb{R}}\|u(\cdot, t+\tau)-u(\cdot, t)\|_{1, q, \mathcal{M}} \mathrm{d} t \quad$ and $\quad T_{2}=\int_{t \in \mathbb{R}}\|u(\cdot, t+\tau)-u(\cdot, t)\|_{-1, r, \mathcal{M}} \mathrm{d} t$.

We have, for $T_{1}$ :

$$
T_{1} \leq \int_{t \in \mathbb{R}}\|u(\cdot, t+\tau)\|_{1, q, \mathcal{M}} \mathrm{d} t+\int_{t \in \mathbb{R}}\|u(\cdot, t)\|_{1, q, \mathcal{M}} \mathrm{d} t \leq 2 C .
$$

By Identity (3.3), we get for $T_{2}$ :

$$
\begin{aligned}
T_{2} & \leq \int_{t \in \mathbb{R}}\left\|\sum_{n \in \mathbb{Z}} \chi_{\tau}^{n}(t)\left(u^{n+1}-u^{n}\right)\right\|_{-1, r, \mathcal{M}} \mathrm{d} t \\
& \left.=\int_{t \in \mathbb{R}} \| \sum_{n \in \mathbb{Z}} \delta t \chi_{\tau}^{n}(t) \partial_{t, \mathcal{D}}(u)^{n+1}\right) \|_{-1, r, \mathcal{M}} \mathrm{d} t .
\end{aligned}
$$

By the triangle inequality and Relation (3.4), we thus obtain:

$$
T_{2} \leq \int_{t \in \mathbb{R}} \sum_{n \in \mathbb{Z}} \delta t \chi_{\tau}^{n}(t)\left\|\partial_{t, \mathcal{D}}(u)^{n+1}\right\|_{-1, r, \mathcal{M}} \mathrm{d} t=\tau \sum_{n \in \mathbb{Z}} \delta t\left\|\partial_{t, \mathcal{D}}(u)^{n}\right\|_{-1, r, \mathcal{M}}=\tau C .
$$

Gathering the estimates of $T_{1}$ and $T_{2}$ yields:

$$
\int_{t \in \mathbb{R}}\|\bar{u}(\cdot, t+\tau)-\bar{u}(\cdot, t)\|_{L^{1}\left(\mathbb{R}^{d}\right)} \mathrm{d} t \leq C|\Omega|^{1-1 / q}(2 \alpha+\tau C(\alpha)),
$$

and it is now easy to verify that the choice of $\alpha$ and $\tau$ suggested by the statement of the lemma yields the desired inequality. The case of negative $\tau$ follows by remarking that, by a change of variable in the integration over time, $\|\bar{u}(\cdot, t+\tau)-\bar{u}(\cdot, t)\|_{L^{1}\left(\mathbb{R}^{d} \times \mathbb{R}\right)}=\|\bar{u}(\cdot, t)-\bar{u}(\cdot, t+|\tau|)\|_{\mathrm{L}^{1}\left(\mathbb{R}^{d} \times \mathbb{R}\right)} \cdot$ 
3.3. A discrete Aubin-Simon's lemma. We are now in position to prove the following compactness result.

Theorem 3.1. Let $\left(u^{k}\right)_{k \in \mathbb{N}}$ be a sequence of discrete functions, i.e. a sequence of functions such that, for $k \in \mathbb{N}, u^{k}$ is a function of a space $\mathrm{H}_{\mathcal{D}}^{k}$ associated to a mesh $\mathcal{M}^{k}$ and a time step $\delta t^{k}$. We suppose that the sequence of meshes $\left(\mathcal{M}^{k}\right)_{k \in \mathbb{N}}$ is regular, in the sense that any $\mathcal{M}^{k}, k \in \mathbb{N}$, satisfies the regularity assumption (2.3) with a unique (i.e. independent of $k$ ) positive real number $\xi$, and that both $h_{\mathcal{M}^{k}}$ and $\delta t^{k}$ tend to zero when $k$ tends to $+\infty$. We suppose that there exists three real numbers $C>0, q \geq 1$ and $r \geq 1$ such that:

$$
\forall k \in \mathbb{N}, \quad \sum_{n=1}^{N^{k}} \delta t^{k}\left\|\left(u^{k}\right)^{n}\right\|_{1, q, \mathcal{M}} \leq C, \quad \sum_{n=2}^{N^{k}} \delta t^{k}\left\|\left(\partial_{t, \mathcal{D}}(u)^{k}\right)^{n}\right\|_{-1, r, \mathcal{M}} \leq C .
$$

Then, up to the extraction of a subsequence, the sequence $\left(u^{k}\right)_{k \in \mathbb{N}}$ converges in $\mathrm{L}^{1}(\Omega \times(0, T))$ to a function $u \in \mathrm{L}^{1}\left(0, T ; \mathrm{W}_{0}^{1, q}\right)$.

Proof. First, we remark that, by Lemma 2.2, there exists a real number $C_{1}$ only depending on the domain $\Omega$, on the parameter $\xi$ characterizing the regularity of the meshes and on $q$ such that, $\forall k \in \mathbb{N}, \forall v \in \mathrm{H}_{\mathcal{M}}^{k}$ (the discrete space associated to $\left.\mathcal{M}^{k}\right),\|v\|_{\mathrm{L}^{1}(\Omega)} \leq C_{1}\|v\|_{1, q, \mathcal{M}}$. Consequently, we get:

$$
\forall k \in \mathbb{N}, \quad\left\|u^{k}\right\|_{\mathrm{L}^{1}(\Omega \times(0, T))} \leq C_{1} C .
$$

Let $\varphi$ be a continuously differentiable function from $\mathbb{R}$ to $[0,1]$, such that $\varphi=1$ on $[0, T]$ and $\varphi$ is equal to zero on $(-\infty,-T) \cup(2 T,+\infty)$. For a given $k \in \mathbb{N}$, let us build a sequence $\left(\left(\hat{u}^{k}\right)^{n}\right)_{n \in \mathbb{Z}}$ of functions of $\mathrm{H}_{\mathcal{M}}^{k}$ as follows:

$$
\begin{array}{|ll}
\text { for } n<-N, & \left(\hat{u}^{k}\right)^{n}=0, \\
\text { for }-N \leq n<0, & \left(\hat{u}^{k}\right)^{n}=\varphi\left(t^{n}\right)\left(u^{k}\right)^{-n}, \\
\text { for } 0 \leq n<N, & \left(\hat{u}^{k}\right)^{n}=\varphi\left(t^{n}\right)\left(u^{k}\right)^{n}=\left(u^{k}\right)^{n}, \\
\text { for } N \leq n \leq 2 N, & \left(\hat{u}^{k}\right)^{n}=\varphi\left(t^{n}\right)\left(u^{k}\right)^{2 N-n}, \\
\text { for } n>2 N, & \left(\hat{u}^{k}\right)^{n}=0,
\end{array}
$$

where we have defined $\left(u^{k}\right)^{0}$ (which does not appear in the statement of the theorem) as $\left(u^{k}\right)^{0}=\left(u^{k}\right)^{1}$. Then we denote by $\bar{u}^{k}$ the function of $\overline{\mathrm{H}}_{\mathcal{D}}$ (so defined over $\left.\mathbb{R}^{d} \times \mathbb{R}\right)$ obtained from the sequence $\left(\left(\hat{u}^{k}\right)^{n}\right)_{n \in \mathbb{Z}}$. The function $\bar{u}^{k}$ is equal to $u^{k}$ on $\Omega \times(0, T)$. Since the function $|\varphi|$ is bounded by 1 , we easily get:

$$
\left\|\bar{u}^{k}\right\|_{L^{1}\left(\mathbb{R}^{d} \times \mathbb{R}\right)} \leq 3\left\|u^{k}\right\|_{L^{1}(\Omega \times(0, T))} \leq 3 C_{1} C, \quad \sum_{n \in \mathbb{Z}} \delta t\left\|\left(u^{k}\right)^{n}\right\|_{1, q, \mathcal{M}} \leq 3 C .
$$

In addition, we have, for $0 \leq n \leq N-1$ :

$$
\begin{aligned}
\partial_{t, \mathcal{D}}\left(\bar{u}^{k}\right)^{-n} & =\frac{\varphi\left(t^{-n}\right)\left(\bar{u}^{k}\right)^{n}-\varphi\left(t^{-n-1}\right)\left(\bar{u}^{k}\right)^{n+1}}{\delta t} \\
& =-\varphi\left(t^{-n-1}\right) \partial_{t, \mathcal{D}}\left(\bar{u}^{k}\right)^{n+1}-\left(\bar{u}^{k}\right)^{n} \frac{\varphi\left(t^{-n-1}\right)-\varphi\left(t^{-n}\right)}{\delta t} .
\end{aligned}
$$

Thus, for any $1 \leq s \leq r$, denoting by $s^{\prime}$ either $s^{\prime}=1-1 / s$ if $s>1$, or $s^{\prime}=+\infty$ if $s=1$ :

$$
\left\|\partial_{t, \mathcal{D}}\left(\bar{u}^{k}\right)^{-n}\right\|_{-1, s, \mathcal{M}}=\sup _{v \in \mathrm{H}_{\mathcal{M}}} \frac{1}{\|v\|_{1, s^{\prime}, \mathcal{M}}} \int_{\Omega} \partial_{t, \mathcal{D}}\left(\bar{u}^{k}\right)^{-n} v \mathrm{~d} \boldsymbol{x}=T_{1}+T_{2},
$$


with:

$$
\begin{aligned}
T_{1} & =\sup _{v \in \mathrm{H}_{\mathcal{M}}} \frac{1}{\|v\|_{1, s^{\prime}, \mathcal{M}}} \int_{\Omega} \varphi\left(t^{-n-1}\right) \partial_{t, \mathcal{D}}\left(u^{k}\right)^{n+1} v \mathrm{~d} \boldsymbol{x}, \\
T_{2} & =\sup _{v \in \mathrm{H}_{\mathcal{M}}} \frac{1}{\|v\|_{1, s^{\prime}, \mathcal{M}}} \int_{\Omega}\left(u^{k}\right)^{n} \frac{\varphi\left(t^{-n-1}\right)-\varphi\left(t^{-n}\right)}{\delta t} v \mathrm{~d} \boldsymbol{x} .
\end{aligned}
$$

We have for $T_{1}$ :

$$
\begin{aligned}
T_{1} & =\varphi\left(t^{-n-1}\right) \sup _{v \in \mathrm{H}_{\mathcal{M}}} \frac{1}{\|v\|_{1, s^{\prime}, \mathcal{M}}} \int_{\Omega} \partial_{t, \mathcal{D}}\left(u^{k}\right)^{n+1} v \mathrm{~d} \boldsymbol{x} \\
& \leq \sup _{v \in \mathrm{H}_{\mathcal{M}}} \frac{1}{\|v\|_{1, s^{\prime}, \mathcal{M}}} \int_{\Omega} \partial_{t, \mathcal{D}}\left(u^{k}\right)^{n+1} v \mathrm{~d} \boldsymbol{x}=\left\|\partial_{t, \mathcal{D}}\left(u^{k}\right)^{n+1}\right\|_{-1, s, \mathcal{M}},
\end{aligned}
$$

which is controlled by $\left\|\partial_{t, \mathcal{D}}\left(u^{k}\right)^{n+1}\right\|_{-1, r, \mathcal{M}}$ thanks to Inequality (2.2). The term $T_{2}$ satisfies:

$$
T_{2} \leq\left\|\varphi^{\prime}\right\|_{L^{\infty}(\mathbb{R})} \sup _{v \in \mathrm{H}_{\mathcal{M}}} \frac{1}{\|v\|_{1, s^{\prime}, \mathcal{M}}} \int_{\Omega}\left(u^{k}\right)^{n} v \mathrm{~d} \boldsymbol{x} .
$$

By Lemma 2.2, the $\mathrm{L}^{d /(d-1)}(\Omega)$-norm is controlled by the $\|\cdot\|_{1, q, \mathcal{M}}$-norm, and we can choose $s$ small enough so that the $\|\cdot\|_{1, s^{\prime}, \mathcal{M}}$-norm controls the $\mathrm{L}^{\tilde{d}}(\Omega)$-norm, where $\tilde{d}$ is defined by $(1 / \tilde{d})+((d-1) / d)=1$. We then get, by Hölder's inequality:

$$
T_{2} \leq C_{2}\left\|\varphi^{\prime}\right\|_{L^{\infty}(\mathbb{R})}\left\|\left(u^{k}\right)^{n}\right\|_{1, q, \mathcal{M}}
$$

where $C_{2}$ only depends on $\Omega, \xi, s$ and $q$. Applying similar arguments for $N \leq n \leq$ $2 N$ yields:

$$
\sum_{n \in \mathbb{Z}} \delta t^{k} \|\left(\partial_{t, \mathcal{D}}\left(\bar{u}^{k}\right)^{n} \|_{-1, s, \mathcal{M}} \leq C_{3}\right.
$$

where $C_{3}$ only depends on $\varphi, \Omega, \xi, s, q$ and $C$.

We may now apply Lemmas 3.2 and 3.4 to obtain that, for any $\epsilon>0$, there exists $\tau_{0}$ only depending on $\Omega, \xi C$ and $\epsilon$ such that:

$$
\forall \tau \text { such that }|\tau| \leq \tau_{0}, \forall k \in \mathbb{N}, \quad\left\|\bar{u}^{k}(\cdot, t+\tau)-\bar{u}^{k}(\cdot, t)\right\|_{\mathrm{L}^{1}\left(\mathbb{R}^{d} \times \mathbb{R}\right)} \leq \epsilon .
$$

In addition, from Lemma 2.3, we have:

$\forall k \in \mathbb{N}, \forall n \in \mathbb{Z}, \forall \boldsymbol{y} \in \mathbb{R}^{d}, \quad\left\|\left(\bar{u}^{k}\right)^{n}(\cdot+\boldsymbol{y})-\left(\bar{u}^{k}\right)^{n}(\cdot)\right\|_{\mathrm{L}^{1}\left(\mathbb{R}^{d}\right)} \leq \sqrt{d}|\boldsymbol{y}|\left\|\left(\bar{u}^{k}\right)^{n}\right\|_{1,1, \mathcal{M}}$.

Multiplying by $\delta t^{k}$ and summing over the time steps yields:

$$
\begin{aligned}
& \forall k \in \mathbb{N}, \forall \boldsymbol{y} \in \mathbb{R}^{d}, \\
& \quad\left\|\left(\bar{u}^{k}\right)^{n}(\cdot+\boldsymbol{y})-\left(\bar{u}^{k}\right)^{n}(\cdot)\right\|_{\mathrm{L}^{1}\left(\mathbb{R}^{d} \times \mathbb{R}\right)} \leq \sqrt{d}|\boldsymbol{y}| \sum_{n \in \mathbb{Z}} \delta t^{k}\left\|\left(\bar{u}^{k}\right)^{n}\right\|_{1,1, \mathcal{M}} .
\end{aligned}
$$

Since, $\forall k \in \mathbb{N}, \bar{u}^{k}$ vanishes outside $\Omega \times(-T, 2 T)$, Hölder's inequality (2.1) yields:

$$
\sum_{n \in \mathbb{Z}} \delta t^{k}\left\|\left(\bar{u}^{k}\right)^{n}\right\|_{1,1, \mathcal{M}} \leq(d|\Omega|)^{1 / q^{\prime}} \sum_{n \in \mathbb{Z}} \delta t^{k}\left\|\left(\bar{u}^{k}\right)^{n}\right\|_{1, q, \mathcal{M}},
$$

which shows that space translates also are uniformly controlled. Kolmogorov's Theorem (e.g. [12, Theorem 14.1 p.833]) thus shows that the sequence $\left(u^{k}\right)_{k \in \mathbb{N}}$ is relatively compact in $\mathrm{L}^{1}(\Omega \times(0, T))$. The regularity of the limit of subsequences is a consequence of the bound on the space translates, namely the fact that the right hand side of (3.7) is linear with respect to $|\boldsymbol{y}|$ (see [13, Section 5.2.2]). 
Remark 3.5 (Regularity of the limit). When the functions of the sequence are more regular than in the assumption of Theorem 3.1, so may be also the limit. For instance, if we suppose:

$$
\forall k \in \mathbb{N}, \quad \sum_{n=1}^{N^{k}} \delta t^{k}\left\|\left(u^{k}\right)^{n}\right\|_{1, q, \mathcal{M}}^{q} \leq C,
$$

then the limit $u$ lies in the space $\mathrm{L}^{q}\left(0, T ; \mathrm{W}_{0}^{1, q}(\Omega)\right)$ (see [13, Section 5.2.2]). This result will be used hereafter.

\section{The SCHEME}

For $\sigma \in \mathcal{E}_{\text {int }}$ and $0 \leq n \leq N$, let $\mathrm{v}_{K, \sigma}^{n+1 / 2}$ be defined by:

$$
\mathrm{v}_{K, \sigma}^{n+1 / 2}=\frac{1}{\delta t} \int_{t^{n}}^{t^{n+1}} \int_{\sigma=K \mid L} \boldsymbol{v}(\boldsymbol{x}, t) \cdot \boldsymbol{n}_{K, \sigma} \mathrm{d} \gamma(\boldsymbol{x}) \mathrm{d} t
$$

The backward first-order in time discretization of (1.1) reads:

$$
\begin{aligned}
& \forall K \in \mathcal{M}, \text { for } 0 \leq n<N \\
& \begin{aligned}
\frac{|K|}{\delta t}\left(u_{K}^{n+1}-u_{K}^{n}\right) & +\sum_{\sigma=K \mid L} \mathrm{v}_{K, \sigma}^{n+1 / 2} u_{\sigma}^{n+1}+\sum_{\sigma=K \mid L} \frac{|\sigma|}{d_{\sigma}}\left(u_{K}^{n+1}-u_{L}^{n+1}\right) \\
& +\sum_{\sigma \in \mathcal{E}(K) \cap \mathcal{E}_{\text {ext }}} \frac{|\sigma|}{d_{\sigma}} u_{K}^{n+1}=\frac{1}{\delta t} \int_{t^{n}}^{t^{n+1}} \int_{K} f(\boldsymbol{x}, t) \mathrm{d} \boldsymbol{x} \mathrm{d} t
\end{aligned}
\end{aligned}
$$

where the approximation of $u$ on an internal edge is given by the usual upwind choice:

$$
\forall \sigma \in \mathcal{E}_{\text {int }}, \sigma=K\left|L, \quad u_{\sigma}^{n+1}=\right| \begin{array}{ll}
u_{K}^{n+1} & \text { if } \mathrm{v}_{K, \sigma} \geq 0, \\
u_{L}^{n+1} & \text { otherwise. }
\end{array}
$$

The initial condition for the scheme is obtained by choosing, for the value of $u^{0}$ over a cell $K \in \mathcal{M}$, the mean value of $u_{0}$ over $K$ :

$$
\forall K \in \mathcal{M}, \quad u_{K}^{0}=\frac{1}{|K|} \int_{K} u_{0}(\boldsymbol{x}) \mathrm{d} \boldsymbol{x} .
$$

\section{Estimates}

Let $\theta \in(1,2)$ and let us define the function $\phi$, from $\mathbb{R}$ to $\mathbb{R}$ by:

$$
\forall y \in \mathbb{R}, \quad \phi^{\prime}(y)=\int_{0}^{y} \frac{1}{1+|s|^{\theta}} \mathrm{d} s, \quad \text { and } \quad \phi(y)=\int_{0}^{y} \phi^{\prime}(s) \mathrm{d} s .
$$

The function $\phi$ enjoys the following features:

(1) the function $\phi^{\prime}$ is positive over $\mathbb{R}^{+}$, negative over $\mathbb{R}^{-}$, and increasing over $\mathbb{R}$; the function $\phi$ is positive and convex over $\mathbb{R}$.

(2) the function $\left|\phi^{\prime}\right|$ is bounded over $\mathbb{R}$; precisely speaking, we have:

$$
\forall y \in \mathbb{R}, \quad\left|\phi^{\prime}(y)\right| \leq \int_{0}^{1} \mathrm{~d} s+\int_{1}^{+\infty} \frac{1}{s^{\theta}} \mathrm{d} s=1+\frac{1}{\theta-1} .
$$


(3) Relation (5.2) yields:

$$
\forall y \in \mathbb{R}, \quad \phi(y) \leq\left(1+\frac{1}{\theta-1}\right)|y| .
$$

In addition, if we denote by $C_{\phi}$ the positive real number defined by $C_{\phi}=$ $\min \left(\phi(1), \phi^{\prime}(1)\right)$, we get, by convexity of $\phi$ :

$$
\forall y \text { such that }|y| \geq 1, \quad \phi(y) \geq C_{\phi}|y| .
$$

In addition, since, for $s \in[0,1]$ and $\theta \in(1,2), 1+|s|^{\theta} \leq 2$, we easily get that $C_{\phi} \geq 1 / 4$.

This function $\phi$ is used in the proof of the following stability result.

Lemma 5.1. Let $u \in \mathrm{H}_{\mathcal{D}}$ be the solution to the scheme (4.2)-(4.4), and $\phi$ be the real function defined over $\mathbb{R}$ by (5.1). Then the following bound holds for $1 \leq M \leq N$ :

$$
\begin{gathered}
\left\|u^{M}\right\|_{\mathrm{L}^{1}(\Omega)}+\sum_{n=1}^{M} \delta t \sum_{\substack{\sigma \in \mathcal{E}_{\text {int }}, \sigma=K \mid L}} \frac{|\sigma|}{d_{\sigma}}\left(u_{K}^{n}-u_{L}^{n}\right)\left[\phi^{\prime}\left(u_{K}^{n}\right)-\phi^{\prime}\left(u_{L}^{n}\right)\right] \\
+\sum_{n=1}^{M} \delta t \sum_{\substack{\sigma \in \mathcal{E}_{\text {ext }}, \sigma \in \mathcal{E}(K)}} \frac{|\sigma|}{d_{\sigma}} u_{K}^{n} \phi^{\prime}\left(u_{K}^{n}\right) \leq C,
\end{gathered}
$$

where $C$ only depends on $\Omega, f, u_{0}$ and $\theta$. Since, for any $\theta \in(1,2), s \phi^{\prime}(s) \geq 0$ for $s \in \mathbb{R}$ and $\phi^{\prime}$ is an increasing function over $\mathbb{R}$, this inequality provides a bound independent of $\theta$ for $u$ in $\mathrm{L}^{\infty}\left(0, T ; \mathrm{L}^{1}(\Omega)\right)$.

Proof. Let us take $\phi^{\prime}\left(u^{n+1}\right)$ as test-function in the scheme, i.e. multiply (4.2) by $\phi^{\prime}\left(u_{K}^{n+1}\right)$ and sum over the control volumes. We get $T_{c}^{n+1}+T_{d}^{n+1}=T_{f}^{n+1}$ with:

$$
\begin{aligned}
T_{c}^{n+1} & =\sum_{K \in \mathcal{M}} \phi^{\prime}\left(u_{K}^{n+1}\right)\left[\frac{|K|}{\delta t}\left(u_{K}^{n+1}-u_{K}^{n}\right)+\sum_{\sigma=K \mid L} \mathrm{v}_{K, \sigma}^{n+1 / 2} u_{\sigma}^{n+1}\right], \\
T_{d}^{n+1} & =\sum_{K \in \mathcal{M}} \phi^{\prime}\left(u_{K}^{n+1}\right)\left[\sum_{\sigma=K \mid L} \frac{|\sigma|}{d_{\sigma}}\left(u_{K}^{n+1}-u_{L}^{n+1}\right)+\sum_{\sigma \in \mathcal{E}(K) \cap \mathcal{E}_{\text {ext }}} \frac{|\sigma|}{d_{\sigma}} u_{K}^{n+1}\right], \\
T_{f}^{n+1} & =\frac{1}{\delta t} \sum_{K \in \mathcal{M}} \phi^{\prime}\left(u_{K}^{n+1}\right) \int_{t^{n}}^{t^{n+1}} \int_{K} f(\boldsymbol{x}, t) \mathrm{d} \boldsymbol{x} \mathrm{d} t .
\end{aligned}
$$

Since the advection field $\boldsymbol{v}$ is divergence-free, by the definition (4.1), we get:

$$
\forall K \in \mathcal{M}, \quad \sum_{\sigma=K \mid L} \mathrm{v}_{K, \sigma}^{n+1 / 2}=0 .
$$

Thanks to Proposition B.1 of Appendix B applied with $\rho_{K}=\rho_{K}^{*}=1, \forall K \in \mathcal{M}$, we thus obtain:

$$
T_{c}^{n+1} \geq \sum_{K \in \mathcal{M}} \frac{|K|}{\delta t}\left[\phi\left(u_{K}^{n+1}\right)-\phi\left(u_{K}^{n}\right)\right] .
$$


Reordering the summation in $T_{d}^{n+1}$, we have:

$$
\begin{gathered}
T_{d}^{n+1}=\sum_{\substack{\sigma \in \mathcal{E}_{\text {int }}, \sigma=K \mid L}} \frac{|\sigma|}{d_{\sigma}}\left(u_{K}^{n+1}-u_{L}^{n+1}\right)\left[\phi^{\prime}\left(u_{K}^{n+1}\right)-\phi^{\prime}\left(u_{L}^{n+1}\right)\right] \\
+\sum_{\substack{\sigma \in \mathcal{E}_{\text {ext }}, \sigma \in \mathcal{E}(K)}} \frac{|\sigma|}{d_{\sigma}} u_{K}^{n+1} \phi^{\prime}\left(u_{K}^{n+1}\right) .
\end{gathered}
$$

Finally, since $\phi$ is bounded over $\mathbb{R}^{+}$by Relation (5.2), we get:

$$
T_{f}^{n+1} \leq \frac{1}{\delta t}\left(1+\frac{1}{\theta-1}\right) \int_{t^{n}}^{t^{n+1}} \int_{\Omega} f(\boldsymbol{x}, t) \mathrm{d} \boldsymbol{x} \mathrm{d} t
$$

Multiplying by $\delta t$ and summing from $n=0$ to $n=M-1$, we thus get:

$$
\begin{array}{r}
\sum_{K \in \mathcal{M}}|K| \phi\left(u_{K}^{M}\right)+\sum_{n=1}^{M} \delta t \sum_{\substack{\sigma \in \mathcal{E}_{\text {int }}, \sigma=K \mid L}} \frac{|\sigma|}{d_{\sigma}}\left(u_{K}^{n}-u_{L}^{n}\right)\left[\phi^{\prime}\left(u_{K}^{n}\right)-\phi^{\prime}\left(u_{L}^{n}\right)\right] \\
+\sum_{n=1}^{M} \delta t \sum_{\substack{\sigma \in \mathcal{E}_{\text {ext }}, \sigma \in \mathcal{E}(K)}} \frac{|\sigma|}{d_{\sigma}} u_{K}^{n} \phi^{\prime}\left(u_{K}^{n}\right) \leq \sum_{K \in \mathcal{M}}|K| \phi\left(u_{K}^{0}\right) \\
+\left(1+\frac{1}{\theta-1}\right) \int_{0}^{t^{M}} \int_{\Omega} f(\boldsymbol{x}, t) \mathrm{d} \boldsymbol{x} \mathrm{d} t,
\end{array}
$$

which concludes the proof thanks to the definition (4.4) of $u^{0}$, Inequality (5.3) and Inequality (5.4).

The following lemma is a central argument of estimates in the elliptic case. It may be found in [16], and is recalled here, together with its proof, for the sake of completeness.

Lemma 5.2. Let $v$ be a function of $\mathrm{H}_{\mathcal{M}}$ and $\phi$ be the real function defined over $\mathbb{R}$ by (5.1). Let $T_{d}(v)$ be given by:

$$
T_{d}(v)=\sum_{\substack{\sigma \in \mathcal{E}_{\text {int }}, \sigma=K \mid L}} \frac{|\sigma|}{d_{\sigma}}\left(v_{K}-v_{L}\right)\left[\phi^{\prime}\left(v_{K}\right)-\phi^{\prime}\left(v_{L}\right)\right]+\sum_{\substack{\sigma \in \mathcal{E}_{\text {ext }}, \sigma \in \mathcal{E}(K)}} \frac{|\sigma|}{d_{\sigma}} v_{K} \phi^{\prime}\left(v_{K}\right) .
$$

Then the following bound holds for $1 \leq p<2$ :

$$
\|v\|_{1, p, \mathcal{M}}^{p} \leq\left[T_{d}(v)\right]^{p / 2}\left[C_{1}+C_{2}\|v\|_{\mathrm{L}^{\theta p /(2-p)}(\Omega)}^{\theta p / 2}\right]
$$

where $C_{1}$ and $C_{2}$ only depend on $p$ and on the regularity of the mesh, i.e. on the parameter $\xi_{\mathcal{M}}$ defined by $(2.3)$.

Proof. Let us first introduce some notations. For any face $\sigma \in \mathcal{E}$ and any function $v \in \mathrm{H}_{\mathcal{M}}$, we define:

$$
\partial_{\sigma} v=\mid \begin{array}{cl}
\frac{v_{K}-v_{L}}{d_{\sigma}} & \text { if } \sigma \in \mathcal{E}_{\text {int }}, \sigma=K \mid L \\
\frac{v_{K}}{d_{\sigma}} & \text { if } \sigma \in \mathcal{E}_{\text {ext }}, \sigma \in \mathcal{E}(K),
\end{array}
$$


and:

$$
a_{\sigma}=\mid \begin{array}{cl}
\frac{\phi^{\prime}\left(v_{K}\right)-\phi^{\prime}\left(v_{L}\right)}{v_{K}-v_{L}} & \text { if } \sigma \in \mathcal{E}_{\mathrm{int}}, \sigma=K \mid L, \\
\frac{\phi^{\prime}\left(v_{K}\right)}{v_{K}} & \text { if } \sigma \in \mathcal{E}_{\mathrm{ext}}, \sigma \in \mathcal{E}(K) .
\end{array}
$$

Note that, for $\sigma \in \mathcal{E}$, the quantity $a_{\sigma}$ is non-negative. With these notations, we have:

$$
\|v\|_{1, p, \mathcal{M}}^{p}=\sum_{\sigma \in \mathcal{E}}|\sigma| d_{\sigma}\left|\partial_{\sigma} v\right|^{p} \quad \text { and } \quad T_{d}(v)=\sum_{\sigma \in \mathcal{E}}|\sigma| d_{\sigma} a_{\sigma}\left(\partial_{\sigma} v\right)^{2} .
$$

By Hölder's inequality, we get:

$$
\|v\|_{1, p, \mathcal{M}}^{p} \leq\left[\sum_{\sigma \in \mathcal{E}}|\sigma| d_{\sigma} a_{\sigma}\left(\partial_{\sigma} v\right)^{2}\right]^{p / 2}\left[\sum_{\sigma \in \mathcal{E}}|\sigma| d_{\sigma} a_{\sigma}^{-p /(2-p)}\right]^{(2-p) / 2} .
$$

For $\sigma \in \mathcal{E}_{\text {int }}, \sigma=K \mid L$, there exists $\bar{v}_{\sigma}$ lying between $v_{K}$ and $v_{L}$ such that $a_{\sigma}=$ $\phi^{\prime \prime}\left(\bar{v}_{\sigma}\right)$. From the expression of $\phi$, we thus get:

$$
\forall \sigma \in \mathcal{E}_{\mathrm{int}}, \sigma=K \mid L, \quad \frac{1}{a_{\sigma}} \leq 1+\max \left(\left|v_{K}\right|,\left|v_{L}\right|\right)^{\theta} .
$$

By a similar argument, we also have:

$$
\forall \sigma \in \mathcal{E}_{\text {ext }}, \sigma \in \mathcal{E}(K), \quad \frac{1}{a_{\sigma}} \leq 1+\left|v_{K}\right|^{\theta} .
$$

Inequality (5.6) thus yields $\|v\|_{1, p, \mathcal{M}}^{p} \leq T_{d}(v)^{p / 2} T_{l}^{(2-p) / 2}$ with:

$$
T_{l}=\sum_{\substack{\sigma \in \mathcal{E}_{\text {int }}, \sigma=K \mid L}}|\sigma| d_{\sigma}\left(1+\max \left(\left|v_{K}\right|,\left|v_{L}\right|\right)^{\theta}\right)^{p /(2-p)}+\sum_{\substack{\sigma \in \mathcal{E}_{\text {ext }}, \sigma \in \mathcal{E}(K)}}|\sigma| d_{\sigma}\left(1+\left|v_{K}\right|^{\theta}\right)^{p /(2-p)} .
$$

Using the inequality $(a+b)^{\alpha} \leq 2^{\alpha}\left(a^{\alpha}+b^{\alpha}\right)$ valid for any positive real numbers $a$, $b$ and $\alpha$, we get:

$$
\begin{aligned}
2^{-p /(2-p)} T_{l} \leq \sum_{\sigma \in \mathcal{E}}|\sigma| d_{\sigma}+ & \sum_{\substack{\sigma \in \mathcal{E}_{\text {int }}, \sigma=K \mid L}}|\sigma| d_{\sigma} \max \left(\left|v_{K}\right|,\left|v_{L}\right|\right)^{\theta p /(2-p)} \\
& +\sum_{\substack{\sigma \in \mathcal{E}_{\text {ext }}, \sigma \in \mathcal{E}(K)}}|\sigma| d_{\sigma}\left|v_{K}\right|^{\theta p /(2-p)} .
\end{aligned}
$$

Remarking that, for any $K \in \mathcal{M}$, the total weight of the term $\left|v_{K}\right|^{\theta p /(2-p)}$ in the last two sums (summing all its occurrences) is at most equal to $\sum_{\sigma \in \mathcal{E}(K)}|\sigma| d_{\sigma}$ and that this quantity is bounded by $C|K|$, with $C$ only depending on the regularity of the mesh, we get:

$$
2^{-p /(2-p)} T_{l} \leq d|\Omega|+C \sum_{K \in \mathcal{M}}|K| v_{K}^{\theta p /(2-p)}=d|\Omega|+C\|v\|_{L^{\theta p /(2-p)}(\Omega)}^{\theta p /(2-p)},
$$

and thus, finally:

$$
\|v\|_{1, p, \mathcal{M}}^{p} \leq T_{d}(v)^{p / 2}\left[2^{p /(2-p)} d|\Omega|+2^{p /(2-p)} C\|v\|_{L^{\theta p /(2-p)}(\Omega)}^{\theta p /(2-p)}\right]^{(2-p) / 2},
$$

which easily yields the desired inequality. 
We are now in position to prove the following estimate, by a technique which is reminiscent of the method used in [4] for the continuous case.

Proposition 5.3. Let $u \in \mathrm{H}_{\mathcal{D}}$ be the solution to the scheme (4.2)-(4.4). Then the following bound holds for $1 \leq p<(d+2) /(d+1)$ :

$$
\sum_{n=1}^{N} \delta t\left\|u^{n}\right\|_{1, p, \mathcal{M}}^{p} \leq C
$$

where $C$ only depends on $\Omega, T, f, u_{0}, p$ and the regularity of the mesh, i.e. on the parameter $\xi_{\mathcal{M}}$ defined by $(2.3)$.

Proof. In this proof, we denote by $C_{i}$ a positive real number only depending on $\Omega, T, f, u_{0}, p, \theta$ and the parameter $\xi_{\mathcal{M}}$ characterizing the regularity of the mesh. Thanks to Lemma 5.2, we get, for $1 \leq p<2$ :

$$
\sum_{n=1}^{N} \delta t\left\|u^{n}\right\|_{1, p, \mathcal{M}}^{p} \leq \sum_{n=1}^{N} \delta t\left[T_{d}\left(u^{n}\right)\right]^{p / 2}\left[C_{1}+C_{2}\left\|u^{n}\right\|_{\mathrm{L}^{\theta p /(2-p)}(\Omega)}^{\theta p / 2}\right]
$$

Since $p<2$, the discrete Hölder's inequality yields:

$$
\begin{aligned}
\sum_{n=1}^{N} \delta t\left\|u^{n}\right\|_{1, p, \mathcal{M}}^{p} \leq & {\left[\sum_{n=1}^{N} \delta t T_{d}\left(u^{n}\right)\right]^{p / 2} } \\
& {\left[\sum_{n=1}^{N} \delta t\left[C_{1}+C_{2}\left\|u^{n}\right\|_{L^{\theta p /(2-p)}(\Omega)}^{\theta p / 2}\right]^{2 /(2-p)}\right]^{(2-p) / 2} . }
\end{aligned}
$$

From Lemma 5.1, we know that:

$$
\sum_{n=1}^{N} \delta t T_{d}\left(u^{n}\right) \leq C_{3}
$$

Let us now apply the inequality $(a+b)^{\alpha} \leq 2^{\alpha}\left(a^{\alpha}+b^{\alpha}\right)$, valid for $a, b, \alpha \geq 0$, to obtain:

$$
\begin{aligned}
\sum_{n=1}^{N} \delta t\left\|u^{n}\right\|_{1, p, \mathcal{M}}^{p} & \leq C_{3}^{p / 2}\left[\sum_{n=1}^{N} 2^{2 /(2-p)} \delta t C_{1}^{2 /(2-p)}\right. \\
& \left.+\sum_{n=1}^{N} 2^{2 /(2-p)} \delta t\left[C_{2}\left\|u^{n}\right\|_{\mathrm{L}^{\theta p /(2-p)}(\Omega)}^{\theta p / 2}\right]^{2 /(2-p)}\right]^{(2-p) / 2} .
\end{aligned}
$$

This last relation yields the existence of $C_{4}$ and $C_{5}$ such that:

$$
\sum_{n=1}^{N} \delta t\left\|u^{n}\right\|_{1, p, \mathcal{M}}^{p} \leq C_{4}+C_{5}\left[\sum_{n=1}^{N} \delta t\left\|u^{n}\right\|_{L^{\theta p /(2-p)}(\Omega)}^{\theta p /(2-p)}\right]^{(2-p) / 2} .
$$

The discrete Sobolev inequality $\|v\|_{1, p, \mathcal{M}}^{p} \geq C_{6}\|v\|_{\mathrm{L}^{p^{*}}(\Omega)}^{p}$, which holds for any $v \in \mathrm{H}_{\mathcal{M}}$ with $p^{*}=d p /(d-p)$, yields:

$C_{6} \sum_{n=1}^{N} \delta t\left\|u^{n}\right\|_{\mathrm{L}^{p^{*}(\Omega)}}^{p} \leq \sum_{n=1}^{N} \delta t\left\|u^{n}\right\|_{1, p, \mathcal{M}}^{p} \leq C_{4}+C_{5}\left[\sum_{n=1}^{N} \delta t\left\|u^{n}\right\|_{\mathrm{L}^{\theta p /(2-p)}(\Omega)}^{\theta p /(2-p)}\right]^{(2-p) / 2}$. 
The idea to conclude the proof is now to modify the right-hand side of this relation to obtain an inequality of the form:

$$
C_{6} \sum_{n=1}^{N} \delta t\left\|u^{n}\right\|_{\mathrm{L}^{p^{*}}(\Omega)}^{p} \leq \sum_{n=1}^{N} \delta t\left\|u^{n}\right\|_{1, p, \mathcal{M}}^{p} \leq C_{7}+C_{8}\left[\sum_{n=1}^{N} \delta t\left\|u^{n}\right\|_{\mathrm{L}^{p^{*}}(\Omega)}^{p}\right]^{\beta}
$$

with an exponent $\beta<1$, which will yield a control on $\sum_{n=1}^{N} \delta t\left\|u^{n}\right\|_{L^{p^{*}}(\Omega)}^{p}$ and, consequently, on $\sum_{n=1}^{N} \delta t\left\|u^{n}\right\|_{1, p, \mathcal{M}}^{p}$. To this purpose, we first use an interpolation inequality, to bound $\left\|u^{n}\right\|_{L^{\theta p /(2-p)(\Omega)}}$ as a function of (a power of) $\left\|u^{n}\right\|_{L^{p^{*}}(\Omega)}$ and $\left\|u^{n}\right\|_{\mathrm{L}^{1}(\Omega)}$, which is uniformly bounded by Lemma 5.1 ; then an Hölder inequality allows to change the exponent (to $p$ ) of this latter norm. Let us recall the interpolation inequality of interest, valid for $1<r<q$ :

$$
\forall v \in \mathrm{L}^{q}(\Omega), \quad\|v\|_{\mathrm{L}^{r}(\Omega)} \leq\|v\|_{\mathrm{L}^{q}(\Omega)}^{\zeta}\|v\|_{\mathrm{L}^{1}(\Omega)}^{1-\zeta}, \quad \text { with } \zeta=\frac{1-1 / r}{1-1 / q} .
$$

Thanks to this inequality and the fact that, for $0 \leq n \leq N,\left\|u^{n}\right\|_{\mathrm{L}^{1}(\Omega)} \leq C_{9}$, we thus get, denoting $r=\theta p /(2-p)$ :

$$
\begin{array}{r}
{\left[\sum_{n=1}^{N} \delta t\left\|u^{n}\right\|_{\mathrm{L}^{\theta p /(2-p)(\Omega)}}^{\theta p /(2-p)}\right]^{(2-p) / 2} \leq C_{10}\left[\sum_{n=1}^{N} \delta t\left\|u^{n}\right\|_{\mathrm{L}^{p^{*}}(\Omega)}^{\zeta r}\right]^{(2-p) / 2}} \\
\text { with } \quad \zeta=\frac{1-1 / r}{1-1 / p^{*}} .
\end{array}
$$

This inequality is valid if $p^{*}>r$, which is equivalent to $p<(2-\theta) d /(d-\theta)$. We may now apply Hölder's inequality to get:

$$
\left[\sum_{n=1}^{N} \delta t\left\|u^{n}\right\|_{L^{\theta p /(2-p)(\Omega)}}^{\theta p /(2-p)}\right]^{(2-p) / 2} \leq C_{8}\left[\sum_{n=1}^{N} \delta t\left\|u^{n}\right\|_{L^{p^{*}}(\Omega)}^{p}\right]^{(2-p) r \zeta / 2 p}
$$

provided that $\zeta r<p$, which reads:

$$
\frac{1}{p} \frac{r-1}{1-1 / p^{*}}<1
$$

Expliciting the values of $p^{*}$ and $r$ as a function of $\theta$ and $p$, it may be seen that this inequality is valid for:

$$
p<\frac{(2-\theta) d+2}{d+1} .
$$

When this inequality is satisfied, since $1 \leq p<2$, we have $(2-p) / 2<1$ and $(2-p) r \zeta / 2 p<1$, and we are thus able to conclude the proof as announced. For $d=1, d=2$ or $d=3$, we have:

$$
\frac{(2-\theta) d+2}{d+1}<\frac{(2-\theta) d}{d-\theta}
$$

for $\theta$ sufficiently close to one, let us say for $\theta \in\left(1, \theta_{0}\right]$. Let $p \in[1,(d+2) /(d+1))$, and $\theta(p)$ be given by $\theta(p)=\min \left(\theta_{0},\left(\theta_{1}+1\right) / 2\right)$ where $\theta_{1} \in(1,2)$ is defined by:

$$
p=\frac{\left(2-\theta_{1}\right) d+2}{d+1}
$$

Then all the inequalities of this proof are valid for $\theta=\theta(p)$, which yields the desired bound. 
Proposition 5.4. Let $u \in \mathrm{H}_{\mathcal{D}}$ be the solution to the scheme (4.2)-(4.4). Then the following bound holds:

$$
\sum_{n=1}^{N-1} \delta t\left\|\partial_{t, \mathcal{D}}(u)^{n}\right\|_{-1,1, \mathcal{M}} \leq C,
$$

where $C$ only depends on $\Omega, T, f, \boldsymbol{v}, u_{0}$ and the regularity of the mesh, i.e. on the parameter $\xi_{\mathcal{M}}$ defined by $(2.3)$.

Proof. Using the notation (5.5), the scheme reads:

$\forall K \in \mathcal{M}$, for $0 \leq n<N$,

$$
\begin{array}{r}
|K| \partial_{t, \mathcal{D}}(u)_{K}^{n}=-\sum_{\sigma=K \mid L} \mathrm{v}_{K, \sigma}^{n+1 / 2} u_{\sigma}^{n+1}-\sum_{\sigma \in \mathcal{E}(K)}|\sigma| \partial_{\sigma} u^{n+1} \\
+\frac{1}{\delta t} \int_{t^{n}}^{t^{n+1}} \int_{K} f(\boldsymbol{x}, t) \mathrm{d} \boldsymbol{x} \mathrm{d} t .
\end{array}
$$

Let $v \in \mathrm{H}_{\mathcal{M}}$. Multiplying each equation of the scheme by $v_{K}$ and summing over the control volumes, we get:

$$
\int_{\Omega} \partial_{t, \mathcal{D}}(u)^{n} v \mathrm{~d} \boldsymbol{x}=\sum_{K \in \mathcal{M}}|K| \partial_{t, \mathcal{D}}(u)_{K}^{n} v_{K}=T_{1}^{n+1}+T_{2}^{n+1}+T_{3}^{n+1},
$$

with:

$$
\begin{aligned}
T_{1}^{n+1} & =-\sum_{K \in \mathcal{M}} v_{K} \sum_{\sigma=K \mid L} \mathrm{v}_{K, \sigma}^{n+1 / 2} u_{\sigma}^{n+1}, \quad T_{2}^{n+1}=-\sum_{K \in \mathcal{M}} v_{K} \sum_{\sigma \in \mathcal{E}(K)}|\sigma| \partial_{\sigma} u^{n+1}, \\
T_{3}^{n+1} & =\frac{1}{\delta t} \int_{t^{n}}^{t^{n+1}} \int_{\Omega} f(\boldsymbol{x}, t) v(\boldsymbol{x}) \mathrm{d} \boldsymbol{x} \mathrm{d} t .
\end{aligned}
$$

Reordering the sums and supposing, without loss of generality, that any face $\sigma$ is oriented in such a way that $\mathrm{v}_{K, \sigma}^{n+1 / 2} \leq 0$, we get for $T_{1}^{n+1}$ :

$$
T_{1}^{n+1}=-\sum_{\substack{\sigma \in \mathcal{E}_{\text {int }}, \sigma=K \mid L}} \mathrm{v}_{K, \sigma}^{n+1 / 2} u_{K}^{n+1}\left(v_{K}-v_{L}\right),
$$

where, by assumption on the velocity field, $\left|\mathrm{v}_{K, \sigma}^{n+1 / 2}\right| \leq\|\boldsymbol{v}\|_{L^{\infty}(\Omega)}|\sigma|, \forall \sigma \in \mathcal{E}_{\text {int. }}$. By the discrete Cauchy-Schwarz inequality, we thus get:

$$
\left|T_{1}^{n+1}\right| \leq C\left\|u^{n+1}\right\|_{L^{2}(\Omega)}\|v\|_{1,2, \mathcal{M}}
$$

where $C$ only depends on $\boldsymbol{v}$ and the parameter $\xi_{\mathcal{M}}$ governing the regularity of the mesh.

By a similar computation, we get for $T_{2}^{n+1}$ :

$$
\left|T_{2}^{n+1}\right|=\left|\sum_{\sigma \in \mathcal{E}}\right| \sigma\left|d_{\sigma} \partial_{\sigma} u^{n+1} \partial_{\sigma} v\right| \leq\left\|u^{n+1}\right\|_{1,1, \mathcal{M}}\|v\|_{1, \infty, \mathcal{M}}
$$

Finally, the term $T_{3}^{n+1}$ satisfies:

$$
\left|T_{3}^{n+1}\right| \leq \frac{1}{\delta t}\|v\|_{L^{\infty}(\Omega)}\|f\|_{L^{1}\left(\Omega \times\left(t^{n}, t^{n+1}\right)\right)} .
$$


Let $p \in(1,(d+2) /(d+1))$ be such that the discrete $\mathrm{W}^{1, p}(\Omega)$ norm of $u$ controls its $\mathrm{L}^{2}(\Omega)$ norm (which, by Lemma 2.2 ) is indeed possible. Since the $\mathrm{L}^{\infty}$ norm is controlled by the $\|\cdot\|_{1, \infty, \mathcal{M}}$ norm, we get, for any $v \in \mathrm{H}_{\mathcal{M}}$ :

$$
\begin{aligned}
\delta t \int_{\Omega} \partial_{t, \mathcal{D}}(u)^{n} v \mathrm{~d} \boldsymbol{x} & \leq \delta t\left(\left|T_{1}^{n+1}\right|+\left|T_{2}^{n+1}\right|+\left|T_{3}^{n+1}\right|\right) \\
& \leq C\left[\delta t\left\|u^{n+1}\right\|_{1, p, \mathcal{M}}+\|f\|_{\mathrm{L}^{1}\left(\Omega \times\left(t^{n}, t^{n+1}\right)\right)}\right]\|v\|_{1, \infty, \mathcal{M}},
\end{aligned}
$$

which, summing over the time steps and using Proposition 5.3, concludes the proof.

\section{Convergence analysis}

In this section, we prove the following result.

Theorem 6.1. Let $\left(u^{k}\right)_{k \in \mathbb{N}}$ be a sequence of discrete solutions, i.e. a sequence of solutions to the scheme (4.2)-(4.4), with a mesh $\mathcal{M}^{k}$ and a time step $\delta t^{k}$. We suppose that the sequence of meshes $\left(\mathcal{M}^{k}\right)_{k \in \mathbb{N}}$ is regular, in the sense that any $\mathcal{M}^{k}$, $k \in \mathbb{N}$, satisfies the regularity assumption (2.3) with a unique (i.e. independent of $k$ ) positive real number $\xi$, and that both $h_{\mathcal{M}^{k}}$ and $\delta t^{k}$ tend to zero when $k$ tends to $+\infty$.

Then, up to the extraction of a subsequence, the sequence $\left(u^{k}\right)_{k \in \mathbb{N}}$ converges in $\mathrm{L}^{1}(\Omega \times(0, T))$ to a function $u \in \mathrm{L}^{p}\left(0, T ; \mathrm{W}_{0}^{1, p}(\Omega)\right)$, for any $p \in[1,(d+2) /(d+1))$, which is a weak solution to the continuous problem, in the sense of Definition 1.1.

Proof. Thanks to the estimates of Propositions 5.3 and 5.4, Theorem 3.1 applies, and the sequence $\left(u^{k}\right)_{k \in \mathbb{N}}$ is known to converge in $\mathrm{L}^{1}(\Omega \times(0, T))$ to a function $u$ which, thanks to Remark 3.5, belongs to $\mathrm{L}^{p}\left(0, T ; \mathrm{W}_{0}^{1, p}(\Omega)\right)$, for any $p \in[1,(d+$ $2) /(d+1))$. We now show that this function is a weak solution to the continuous problem.

Let $\varphi \in \mathrm{C}_{c}^{\infty}(\Omega \times[0, T))$. For a given discretization $\mathcal{M}^{k}$ and $\delta t^{k}$, we denote by $\varphi_{K}^{n}$ the quantity:

$$
\forall K \in \mathcal{M}^{k}, \text { for } 0 \leq n \leq N^{k}, \quad \varphi_{K}^{n}=\varphi\left(\boldsymbol{x}_{K}, t^{n}\right) .
$$

Let us now multiply by $\varphi_{K}^{n}$ each equation of the scheme, multiply by $\delta t$ and sum over the control volumes and the time steps, to obtain:

$$
T_{\partial t}^{k}+T_{c}^{k}+T_{d}^{k}=T_{f}^{k}
$$

with, dropping for short the superscripts $k$ and using the notation (5.5):

$$
\begin{aligned}
T_{\partial t} & =\sum_{n=0}^{N-1} \sum_{K \in \mathcal{M}}|K|\left(u_{K}^{n+1}-u_{K}^{n}\right) \varphi_{K}^{n}, \\
T_{c} & =\sum_{n=0}^{N-1} \delta t \sum_{K \in \mathcal{M}} \varphi_{K}^{n} \sum_{\sigma=K \mid L} \mathrm{v}_{K, \sigma}^{n+1 / 2} u_{\sigma}^{n+1}, \\
T_{d} & =\sum_{n=0}^{N-1} \delta t \sum_{K \in \mathcal{M}} \varphi_{K}^{n} \sum_{\sigma \in \mathcal{E}(K)}|\sigma| \partial_{\sigma} u^{n+1}, \\
T_{f} & =\sum_{n=0}^{N-1} \sum_{K \in \mathcal{M}} \varphi_{K}^{n} \int_{t^{n}}^{t^{n+1}} \int_{K} f(\boldsymbol{x}, t) \mathrm{d} \boldsymbol{x} \mathrm{d} t .
\end{aligned}
$$


For the first term, we get, reordering the sums:

$$
T_{\partial t}=-\sum_{n=1}^{N} \sum_{K \in \mathcal{M}}|K| u_{K}^{n}\left(\varphi_{K}^{n}-\varphi_{K}^{n-1}\right)-\sum_{K \in \mathcal{M}}|K| u_{K}^{0} \varphi_{K}^{0},
$$

which yields:

$$
T_{\partial t}=-\int_{t=O}^{T} \int_{\Omega} u(\boldsymbol{x}, t) \partial_{t} \varphi(\boldsymbol{x}, t) \mathrm{d} \boldsymbol{x} \mathrm{d} t+R_{1}-\int_{\Omega} u_{0}(\boldsymbol{x}) \varphi(\boldsymbol{x}, 0) \mathrm{d} \boldsymbol{x}+R_{2},
$$

the terms $R_{1}$ and $R_{2}$ being defined below. Using the fact that $u$ is piecewise constant, the first one reads:

$$
R_{1}=-\sum_{n=1}^{N} \delta t \sum_{K \in \mathcal{M}}|K| u_{K}^{n}\left[\frac{\varphi_{K}^{n}-\varphi_{K}^{n-1}}{\delta t}-\frac{1}{|K| \delta t} \int_{t^{n-1}}^{t^{n}} \int_{K} \partial_{t} \varphi(\boldsymbol{x}, t) \mathrm{d} \boldsymbol{x} \mathrm{d} t\right]
$$

so $\left|R_{1}\right| \leq c_{\varphi}(h+\delta t)\|u\|_{\mathrm{L}^{1}(\Omega \times(0, T))}$ where $c_{\varphi}=\|\varphi\|_{\mathrm{W}^{2, \infty}(\Omega \times[0, T))}$. The term $R_{2}$ reads:

$$
R_{2}=-\sum_{K \in \mathcal{M}} \int_{K} u_{0}(\boldsymbol{x})\left[\varphi_{K}^{0}-\varphi(\boldsymbol{x}, 0)\right] \mathrm{d} \boldsymbol{x}
$$

so $\left|R_{2}\right| \leq c_{\varphi} h\left\|u_{0}\right\|_{\mathrm{L}^{1}(\Omega)}$.

We now turn to the convection term, which we write $T_{c}=T_{c, 1}+T_{c, 2}$, with:

$$
\begin{aligned}
T_{c, 1} & =\sum_{n=0}^{N-1} \delta t \sum_{K \in \mathcal{M}} \varphi_{K}^{n} u_{K}^{n+1} \sum_{\sigma=K \mid L} \mathrm{v}_{K, \sigma}^{n+1 / 2}, \\
T_{c, 2} & =\sum_{n=0}^{N-1} \delta t \sum_{K \in \mathcal{M}} \varphi_{K}^{n} \sum_{\sigma=K \mid L} \mathrm{v}_{K, \sigma}^{n+1 / 2}\left(u_{\sigma}^{n+1}-u_{K}^{n+1}\right) .
\end{aligned}
$$

For this proof, we choose not to use the fact that the velocity field is divergencefree, which is useless for the convergence (but has been used for stability). By the definition of $\mathrm{v}_{K, \sigma}^{n+1 / 2}$, the term $T_{c, 1}$ reads:

$$
\begin{aligned}
T_{c, 1} & =\sum_{n=0}^{N-1} \varphi_{K}^{n} \int_{t^{n}}^{t^{n+1}} \int_{K} u(\boldsymbol{x}, t) \operatorname{div} \boldsymbol{v}(\boldsymbol{x}, t) \mathrm{d} \boldsymbol{x} \mathrm{d} t \\
& =\int_{0}^{T} \int_{\Omega} u(\boldsymbol{x}, t) \varphi(\boldsymbol{x}, t) \operatorname{div} \boldsymbol{v}(\boldsymbol{x}, t) \mathrm{d} \boldsymbol{x} \mathrm{d} t+R_{3},
\end{aligned}
$$

with:

$$
R_{3}=\sum_{n=0}^{N-1} \int_{t^{n}}^{t^{n+1}} \int_{K} u(\boldsymbol{x}, t) \operatorname{div} \boldsymbol{v}(\boldsymbol{x}, t)\left[\varphi_{K}^{n}-\varphi(\boldsymbol{x}, t)\right] \mathrm{d} \boldsymbol{x} \mathrm{d} t
$$

so $\left|R_{3}\right| \leq c_{\varphi} h\|\boldsymbol{v}\|_{\mathrm{W}^{1, \infty}(\Omega \times(0, T))}\|u\|_{\mathrm{L}^{1}(\Omega \times(0, T))}$.

We now decompose $T_{c, 2}=T_{c, 3}+R_{4}$ where $T_{c, 3}$ is chosen to be:

$$
\begin{aligned}
T_{c, 3} & =-\sum_{n=0}^{N-1} \sum_{K \in \mathcal{M}} u_{K}^{n+1} \sum_{\sigma=K \mid L} \int_{t^{n}}^{t^{n+1}} \int_{\sigma} \varphi(\boldsymbol{x}, t) \boldsymbol{v}(\boldsymbol{x}, t) \cdot \boldsymbol{n}_{K} \mathrm{~d} \gamma(\boldsymbol{x}) \mathrm{d} t \\
& =-\int_{0}^{T} \int_{\Omega} u(\boldsymbol{x}, t) \operatorname{div}[\varphi(\boldsymbol{x}, t) \boldsymbol{v}(\boldsymbol{x}, t)] \mathrm{d} \boldsymbol{x} \mathrm{d} t
\end{aligned}
$$


and, by difference and reordering the sums, we obtain for $R_{4}$ :

$$
R_{4}=\sum_{n=0}^{N-1} \sum_{\substack{\sigma \in \mathcal{E}_{\text {int }}, \sigma=K \mid L}}\left(u_{K}^{n+1}-u_{L}^{n+1}\right) \int_{t^{n}}^{t^{n+1}} \int_{\sigma}\left[\varphi(\boldsymbol{x}, t)-\varphi_{L}^{n}\right] \boldsymbol{v}(\boldsymbol{x}, t) \cdot \boldsymbol{n}_{K, \sigma} \mathrm{d} \gamma(\boldsymbol{x}) \mathrm{d} t
$$

where, without loss of generality, we have chosen for the faces the orientation such that $\mathrm{v}_{K, \sigma}^{n+1 / 2} \geq 0$. We thus get:

$$
\begin{aligned}
\left|R_{4}\right| & \leq c_{\varphi} h\|\boldsymbol{v}\|_{L^{\infty}(\Omega \times(0, T))} \sum_{n=0}^{N-1} \delta t \sum_{\substack{\sigma \in \mathcal{E}_{\text {int }}, \sigma=K \mid L}}|\sigma| d_{\sigma} \frac{\left|u_{K}^{n+1}-u_{L}^{n+1}\right|}{d_{\sigma}} \\
& =c_{\varphi} h\|\boldsymbol{v}\|_{L^{\infty}(\Omega \times(0, T))} \sum_{n=1}^{N} \delta t\left\|u^{n}\right\|_{1,1, \mathcal{M}} .
\end{aligned}
$$

Let us now turn to the diffusion term. By a standard reordering of the summations, we get:

$$
T_{d}=\sum_{n=0}^{N-1} \delta t \sum_{K \in \mathcal{M}} u_{K}^{n+1} \sum_{\sigma \in \mathcal{E}(K)}|\sigma| \partial_{\sigma} \varphi^{n}
$$

which reads:

$$
\begin{aligned}
T_{d} & =-\sum_{n=0}^{N-1} \sum_{K \in \mathcal{M}} u_{K}^{n+1} \int_{t^{n}}^{t^{n+1}} \int_{K} \Delta \varphi(\boldsymbol{x}, t) \mathrm{d} \boldsymbol{x} \mathrm{d} t+R_{5} \\
& =-\int_{0}^{T} \int_{\Omega} u(\boldsymbol{x}, t) \Delta \varphi(\boldsymbol{x}, t) \mathrm{d} \boldsymbol{x} \mathrm{d} t+R_{5},
\end{aligned}
$$

with:

$$
\begin{aligned}
R_{5}=\sum_{n=0}^{N-1} \delta t \sum_{K \in \mathcal{M}} u_{K}^{n+1} \sum_{\sigma \in \mathcal{E}(K)}|\sigma| R_{K, \sigma}, \\
R_{K, \sigma}=\partial_{\sigma} \varphi^{n}+\frac{1}{|\sigma| \delta t} \int_{t^{n}}^{t^{n+1}} \int_{\sigma} \nabla \varphi(\boldsymbol{x}, t) \cdot \boldsymbol{n}_{K, \sigma} \mathrm{d} \gamma(\boldsymbol{x}) \mathrm{d} t .
\end{aligned}
$$

For any face $\sigma \in \mathcal{E}_{\text {int }}, \sigma=K \mid L$, we have $R_{K, \sigma}=-R_{L, \sigma}$; in addition, for any $K \in \mathcal{M}$ and any $\sigma \in \mathcal{E}(K),\left|R_{K, \sigma}\right| \leq c_{\varphi}(h+\delta t)$. Hence, reordering once again the sums:

so:

$$
R_{5}=\sum_{n=0}^{N-1} \delta t \sum_{\sigma \in \mathcal{E}(K)}|\sigma| d_{\sigma} \partial_{\sigma} u_{n+1} R_{K, \sigma}
$$

$$
\left|R_{5}\right| \leq c_{\varphi}(h+\delta t) \sum_{n=0}^{N-1} \delta t \sum_{\sigma \in \mathcal{E}}|\sigma| d_{\sigma}\left|\partial_{\sigma} u_{n+1}\right|=c_{\varphi}(h+\delta t)\left\|u_{n+1}\right\|_{1,1, \mathcal{M}} .
$$

Finally, we have for the last term:

$$
T_{f}=\int_{0}^{T} \int_{\Omega} f(\boldsymbol{x}, t) \varphi(\boldsymbol{x}, t) \mathrm{d} \boldsymbol{x} \mathrm{d} t+R_{6},
$$

with $\left|R_{6}\right| \leq c_{\varphi}(h+\delta t)$. 
Finally, gathering all the terms and remarking that $-\operatorname{div}(\varphi \boldsymbol{v})+\varphi \operatorname{div}(\boldsymbol{v})=-\boldsymbol{v} \cdot \nabla \varphi$, we get:

$$
\begin{array}{r}
-\int_{t=O}^{T} \int_{\Omega} u(\boldsymbol{x}, t) \partial_{t} \varphi(\boldsymbol{x}, t) \mathrm{d} \boldsymbol{x} \mathrm{d} t-\int_{\Omega} u_{0}(\boldsymbol{x}) \varphi(\boldsymbol{x}, 0) \mathrm{d} \boldsymbol{x} \\
-\int_{0}^{T} \int_{\Omega} u(\boldsymbol{x}, t) \boldsymbol{v}(\boldsymbol{x}, t) \cdot \nabla \varphi(\boldsymbol{x}, t) \mathrm{d} \boldsymbol{x} \mathrm{d} t-\int_{0}^{T} \int_{\Omega} u(\boldsymbol{x}, t) \Delta \varphi(\boldsymbol{x}, t) \mathrm{d} \boldsymbol{x} \mathrm{d} t \\
=\int_{0}^{T} \int_{\Omega} f(\boldsymbol{x}, t) \varphi(\boldsymbol{x}, t) \mathrm{d} \boldsymbol{x} \mathrm{d} t+R
\end{array}
$$

with $|R| \leq C(h+\delta t)$ where $C$ is controlled by the estimates satisfied by the solution, the regularity of $\boldsymbol{v}$ and $\varphi$ and independently of the mesh. Letting $h$ and $\delta t$ tend to zero in this equation and then integrating by parts the diffusion term thanks to the regularity of the limit thus concludes the proof.

\section{Appendix A. A version of the Lions And Aubin-Simon lemma}

\section{A.1. Lions's lemma.}

Lemma A.1. Let $X, B$ and $Y$ be three Banach spaces satisfying the following hypotheses:

(i) $X$ is compactly imbedded into $B$.

(ii) There exists a vectorial space $F$ such that $B$ and $Y$ are imbedded into $F$ and, for all sequence $\left(u_{n}\right)_{n \in \mathbb{N}}$ of $B \cap Y$, if $u_{n} \rightarrow u$ in $B$ and $u_{n} \rightarrow v$ in $Y$, then $u=v$.

Then, for all $\epsilon>0$ there exists $C_{\epsilon} \in \mathbb{R}$ such that $\|u\|_{B} \leq \epsilon\|u\|_{X}+C_{\epsilon}\|u\|_{Y}$, for all $u \in X \cap Y$.

Proof. We perform the proof by contradiction. We assume that there exists $\epsilon>0$ and a sequence $\left(u_{n}\right)_{n \in \mathbb{N}}$ such that $u_{n} \in X \cap Y$ and $1=\left\|u_{n}\right\|_{B}>\epsilon\left\|u_{n}\right\|_{X}+n\left\|u_{n}\right\|_{Y}$, for all $n \in \mathbb{N}$. Then, $\left(u_{n}\right)_{n \in \mathbb{N}}$ is bounded in $X$ and therefore relatively compact in $B$. Thus, we can assume that $u_{n} \rightarrow u$ in $B$ and $\|u\|_{B}=1$. Furthermore $u_{n} \rightarrow 0$ in $Y$ (since $\left.\left\|u_{n}\right\|_{Y} \leq 1 / n\right)$. Then the second hypothesis of Lemma A.1 gives $u=0$, which is in contradiction with $\|u\|_{B}=1$.

Remark A.2. In some practical case, the second hypothesis of Lemma A.1 may be replaced by the following assumption:

$(\text { ii })^{\prime}$ There exists a topological vectorial space $F$ such that $B$ and $Y$ are continuously imbedded into $F$.

Then:

(1) Assumption (ii)' is stronger than Assumption (ii),

(2) Assumption (ii)' implies the existence of a Banach space $G$ such that $B$ and $Y$ are continuously imbedded into $G$,

(3) as a consequence, under Assumption $(\text { ii })^{\prime}$, Lemma A.1 may be proven using directly a classical lemma due to J.L. Lions.

We first prove the first assertion. Then, let $\left(u_{n}\right)_{n \in \mathbb{N}}$ be a sequence of $B \cap Y$ such that $u_{n} \rightarrow u$ in $B$ and $u_{n} \rightarrow v$ in $Y$. Thanks to the continuous imbedding from $B$ and $Y$ in $F$, one has $u_{n} \rightarrow u$ in $F$ and $u_{n} \rightarrow v$ in $F$. Then, $u=v$, which is Assumption $(i i)$. 
We now turn to the second point. We set $G=B+Y$ and for $u \in G$, one sets $\|u\|_{G}=\inf \left\{\left\|u_{1}\right\|_{B}+\left\|u_{2}\right\|_{Y}, u=u_{1}+u_{2}, u_{1} \in B, u_{2} \in Y\right\}$. The only difficulty for proving that $\|\cdot\|_{G}$ is a norm on $G$ is to prove that $\|u\|_{G}=0 \Rightarrow u=0$. Let $u$ be such an element of $G$, i.e. be such that $\|u\|_{G}=0$. There exists a sequence $\left(u_{1, n}\right)_{n \in \mathbb{N}}$ in $B$ and a sequence $\left(u_{2, n}\right)_{n \in \mathbb{N}}$ in $Y$ such that $u=u_{1, n}+u_{2, n}$, for all $n \in \mathbb{N}$ and $u_{1, n} \rightarrow 0$ in $B, u_{2, n} \rightarrow 0$ in $Y$, as $n \rightarrow+\infty$. Thus both sequences tend to zero in $F$, which proves that $u=0$.

Since both $B$ and $Y$ are continuously imbedded in $G$ (since $\|u\|_{B} \leq\|u\|_{G}$ and $\left.\|u\|_{Y} \leq\|u\|_{G}\right)$, which is a Banach space, the proof is complete.

Let us now address the third issue. Lemma A.1 is the Lions lemma if $F=Y$. Otherwise, this latter lemma may be applied with $G$ instead of $Y$ and gives that, for all $\epsilon>0$, there exists $C_{\epsilon} \in \mathbb{R}$ such that $\|u\|_{B} \leq \epsilon\|u\|_{X}+C_{\epsilon}\|u\|_{G}$, for all $u \in B$. Since $\|\cdot\|_{G} \leq\|\cdot\|_{Y}$, we obtain Lemma A.1.

Remark A.3. We give now an example where the hypotheses of Lemma A.1 are satisfied. Let $\Omega$ is a bounded open set of $\mathbb{R}^{d}(d \geq 1)$ with a Lipschitz continuous boundary. Let $1 \leq p, r \leq \infty, X=\mathrm{W}^{1, p}(\Omega), B=\mathrm{L}^{p}(\Omega)$ and $Y=\mathrm{W}^{-1, r}(\Omega)=$ $\left(\mathrm{W}_{0}^{1, r^{\prime}}\right)(\Omega)^{\star}$, with $r^{\prime}=r /(r-1)$, that is the (topological) dual space of $W_{0}^{1, r^{\prime}}(\Omega)$. The first hypothesis of Lemma A.1 is satisfied. For the second hypothesis, we distinguish the cases $r=1$ and $r>1$. In the case $r>1$, it is possible to take $F=\left(\mathrm{C}_{c}^{\infty}(\Omega)\right)^{\prime}$, that is the set of linear applications from $\mathrm{C}_{c}^{\infty}(\Omega)$ to $\mathbb{R}$ (without any continuity requirement). In the case $r=1$, the choice $F=\left(\mathrm{C}_{c}^{\infty}(\Omega)\right)^{\prime}$ is not convenient since $\mathrm{C}_{c}^{\infty}(\Omega)$ is not dense in $\mathrm{W}_{0}^{1, \infty}(\Omega)$ (and therefore two different elements of $W^{-1,1}(\Omega)$ can have the same restriction on $\mathrm{C}_{c}^{\infty}(\Omega)$ ). But, in order to apply Lemma A.1, it is possible to take $F=Y$ since in this case $B$ is imbedded in $Y$ (as usual, one identifies here $u \in B$ with the linear form $\varphi \mapsto \int_{\Omega} u \varphi \mathrm{d} \boldsymbol{x}$, with $\varphi \in \mathrm{C}_{c}^{\infty}(\Omega)$ for the case $r>1$ and $\varphi \in \mathrm{W}_{0}^{1, \infty}(\Omega)$ in the case $\left.r=1\right)$.

\section{A.2. Aubin-Simon's compactness result.}

Lemma A.4. Let $X, B$ and $Y$ be three Banach spaces satisfying the following hypotheses:

(1) $X$ is compactly imbedded into $B$.

(2) There exists a vectorial space $F$ such that $B$ and $Y$ are imbedded into $F$ and, for all sequence $\left(u_{n}\right)_{n \in \mathbb{N}}$ of $B \cap Y$, if $u_{n} \rightarrow u$ in $B$ and $u_{n} \rightarrow v$ in $Y$, then $u=v$.

Let $T>0, p \in[1, \infty]$ and $\left(u_{n}\right)_{n \in \mathbb{N}}$ be a bounded sequence of $\mathrm{L}^{p}((0, T), X)$. Let $q \in[1, \infty]$ and assume that the sequence $\left(\partial_{t} u_{n}\right)_{n \in \mathbb{N}}$ is bounded in $\mathrm{L}^{q}((0, T), Y)$. Then, there exists $u \in L^{r}((0, T), B)$ such that, up to a subsequence, $u_{n} \rightarrow u$ in $L^{r}((0, T), B)$, as $n \rightarrow+\infty$, with $r=\min \{p, q\}$.

Proof. For short, we restrict the exposition to the case where $(i i)^{\prime}$ holds, which allows a simple proof with the classical Aubin-Simon's compactness result. We take $G=B+Y$ with the norm defined in Remark A.2. we obtain that the sequence $\left(\partial_{t} u_{n}\right)_{n \in \mathbb{N}}$ is bounded in $L^{q}((0, T), G)$ and $B$ is continuously imbedded in $G$. Then the Aubin-Simon's compactness lemma gives the desired result.

Remark A.5. We give here some precision on the sense of " $\partial_{t} u \in \mathrm{L}^{q}((0, T), Y)$ ". Let $X$ and $Y$ two Banach spaces and $p, q \in[1, \infty]$. Assuming that $u \in \mathrm{L}^{p}((0, T), X)$, 
the weak derivative of $u$ is defined by its action on test functions, that is its action on $\varphi$ for all $\varphi \in \mathrm{C}_{c}^{\infty}((0, T)$ ) (note that $\varphi$ takes its values in $\mathbb{R})$. Actually, if $\varphi \in \mathrm{C}_{c}^{\infty}((0, T))$, the function $\left(\partial_{t} \varphi\right) u$ belongs to $\mathrm{L}^{p}((0, T), X)$ and therefore to $\mathrm{L}^{1}((0, T), X)$ and the action of $\partial_{t} u$ on $\varphi$ is defined as:

$$
\left\langle\partial_{t} u, \varphi\right\rangle=-\int_{0}^{T} \partial_{t} \varphi(t) u(t) \mathrm{d} t .
$$

Note that $\left\langle\partial_{t} u, \varphi\right\rangle \in X$.

In order to give a sense to " $\partial_{t} u \in \mathrm{L}^{q}((0, T), Y)$ ", we assume (as in Lemma A.1 and Lemma A.4) that $X$ and $Y$ are imbedded in the same vectorial space $F$. Then $\partial_{t} u \in \mathrm{L}^{q}((0, T), Y)$ means that there exists $v \in \mathrm{L}^{q}((0, T), Y)$ (and then $v$ is unique) such that:

$$
-\int_{0}^{T} \partial_{t} \varphi(t) u(t) \mathrm{d} t=\int_{0}^{T} \varphi(t) v(t) \mathrm{d} t \text { for all } \varphi \in \mathrm{C}_{c}^{\infty}((0, T))
$$

this equality making sense since:

$$
\int_{0}^{T} \partial_{t} \varphi(t) u(t) \mathrm{d} t \in X \subset F \quad \text { and } \quad \int_{0}^{T} \varphi(t) v(t) \mathrm{d} t \in Y \subset F .
$$

\section{Appendix B. A Stability Result for a general Class of CONVECTiON OPERATORS}

In a compressible flow, the mass balance reads:

$$
\partial_{t} \rho+\operatorname{div}(\rho \boldsymbol{v})=0 .
$$

Let $\phi$ be a regular real function, and let us suppose that $\rho, z$ and $\boldsymbol{v}$ are regular scalar (for $\rho$ and $z$ ) and vector-valued ( $\boldsymbol{v}$ ) fields, and that $\boldsymbol{v} \cdot \boldsymbol{n}=0$ on $\partial \Omega$. Then we have:

$$
\begin{array}{r}
\int_{\Omega} \phi^{\prime}(z)\left[\partial_{t}(\rho z)+\operatorname{div}(\rho z \boldsymbol{v})\right] \mathrm{d} \boldsymbol{x}=\int_{\Omega} \rho \phi^{\prime}(z) \partial_{t} z+\rho \phi^{\prime}(z) \nabla z \cdot \boldsymbol{v} \mathrm{d} \boldsymbol{x} \\
=\int_{\Omega} \rho \partial_{t}[\phi(z)]+\rho \nabla[\phi(z)] \cdot \boldsymbol{v} \mathrm{d} \boldsymbol{x}=\int_{\Omega} \rho \partial_{t} \phi(z)-\phi(z) \operatorname{div}(\rho \boldsymbol{v}) \mathrm{d} \boldsymbol{x} \\
=\int_{\Omega} \rho \partial_{t} \phi(z)+\phi(z) \partial_{t} \rho \mathrm{d} \boldsymbol{x}=\frac{d}{d t} \int_{\Omega} \rho \phi(z) \mathrm{d} \boldsymbol{x} .
\end{array}
$$

Taking for $z$ one component of the velocity itself and $\phi(s)=s^{2} / 2$, this computation is the central argument of the so-called kinetic energy conservation theorem. If $z$ satisfies $\partial_{t}(\rho z)+\operatorname{div}(\rho z \boldsymbol{v})-\operatorname{div}(\lambda \nabla z)=0, \lambda \geq 0$, choosing $\phi(s)=\min (0, s)^{2}$, using $\phi(z)$ as a test function and applying (B.2) yields the fact that $z$ remains nonnegative, if its initial condition is non-negative (which can also be seen by noting that, thanks to (B.1), we have $\partial_{t}(\rho z)+\operatorname{div}(\rho z \boldsymbol{v})=\rho\left[\partial_{t} z+\boldsymbol{v} \cdot \nabla z\right]$, and this latter operator is known to satisfy a maximum principle).

The aim of this section is to prove a discrete analogue to (B.2). We thus generalize the proofs already given for the specific choices for $\phi$ mentionned above, namely for $\phi(s)=s^{2} / 2$ in [15] and for $\phi(s)=\min (0, s)^{2}$ in [18]. 
Proposition B.1. Let $\left(\rho_{K}\right)_{K \in \mathcal{M}},\left(\rho_{K}^{*}\right)_{K \in \mathcal{M}}$ and $\left(F_{K, \sigma}\right)_{K \in \mathcal{M}, \sigma \in \mathcal{E}(K) \cap \mathcal{E}_{\text {int }}}$ be three families of real numbers such that:

(i) $\forall K \in \mathcal{M}, \rho_{K} \geq 0, \rho_{K}^{*} \geq 0$

(ii) $\forall \sigma \in \mathcal{E}_{\text {int }}, \sigma=K \mid L, F_{K, \sigma}=-F_{L, \sigma}$,

(iii) $\forall K \in \mathcal{M}, \quad \frac{|K|}{\delta t}\left(\rho_{K}-\rho_{K}^{*}\right)+\sum_{\sigma=K \mid L} F_{K, \sigma}=0$.

Let $\phi$ be a real convex function defined over $\mathbb{R}$, and let $\left(z_{K}\right)_{K \in \mathcal{M}}$ and $\left(z_{K}^{*}\right)_{K \in \mathcal{M}}$ be two families of real numbers. For $\sigma \in \mathcal{E}_{\text {int }}, \sigma=K \mid L$, we denote by $z_{\sigma}$ the quantity defined by $z_{\sigma}=z_{K}$ if $F_{K, \sigma} \geq 0$ and $z_{\sigma}=z_{L}$ otherwise. Then:

$$
\begin{aligned}
\sum_{K \in \mathcal{M}} \phi^{\prime}\left(z_{K}\right)\left[\frac{|K|}{\delta t}\left(\rho_{K} z_{K}-\rho_{K}^{*} z_{K}^{*}\right)+\right. & \left.\sum_{\sigma=K \mid L} F_{K, \sigma} z_{\sigma}\right] \\
& \geq \sum_{K \in \mathcal{M}} \frac{|K|}{\delta t}\left[\rho_{K} \phi\left(z_{K}\right)-\rho_{K}^{*} \phi\left(z_{K}^{*}\right)\right] .
\end{aligned}
$$

Proof. Let us write:

$$
\sum_{K \in \mathcal{M}} \phi^{\prime}\left(z_{K}\right)\left[\frac{|K|}{\delta t}\left(\rho_{K} z_{K}-\rho_{K}^{*} z_{K}^{*}\right)+\sum_{\sigma=K \mid L} F_{K, \sigma} z_{\sigma}\right]=T_{1}+T_{2}
$$

with:

$$
T_{1}=\sum_{K \in \mathcal{M}} \frac{|K|}{\delta t} \phi^{\prime}\left(z_{K}\right)\left(\rho_{K} z_{K}-\rho_{K}^{*} z_{K}^{*}\right), \quad T_{2}=\sum_{K \in \mathcal{M}} \phi^{\prime}\left(z_{K}\right)\left[\sum_{\sigma=K \mid L} F_{K, \sigma} z_{\sigma}\right] .
$$

The first term may be split as $T_{1}=T_{1,1}+T_{1,2}$ with:

$$
T_{1,1}=\sum_{K \in \mathcal{M}} \frac{|K|}{\delta t} \phi^{\prime}\left(z_{K}\right) z_{K}\left(\rho_{K}-\rho_{K}^{*}\right), \quad T_{1,2}=\sum_{K \in \mathcal{M}} \frac{|K|}{\delta t} \rho_{K}^{*} \phi^{\prime}\left(z_{K}\right)\left(z_{K}-z_{K}^{*}\right) .
$$

By convexity of $\phi$, we get:

$$
T_{1,2} \geq \sum_{K \in \mathcal{M}} \frac{|K|}{\delta t} \rho_{K}^{*}\left(\phi\left(z_{K}\right)-\phi\left(z_{K}^{*}\right)\right)
$$

The second term reads $T_{2}=T_{2,1}+T_{2,2}+T_{2,3}$ with:

$$
\begin{array}{r}
T_{2,1}=\sum_{K \in \mathcal{M}} \phi^{\prime}\left(z_{K}\right) z_{K}\left[\sum_{\sigma=K \mid L} F_{K, \sigma}\right], \quad T_{2,2}=\sum_{K \in \mathcal{M}} \phi\left(z_{K}\right)\left[-\sum_{\sigma=K \mid L} F_{K, \sigma}\right] \\
T_{2,3}=\sum_{K \in \mathcal{M}}\left[\sum_{\sigma=K \mid L} F_{K, \sigma}\left(\phi\left(z_{K}\right)+\phi^{\prime}\left(z_{K}\right)\left(z_{\sigma}-z_{K}\right)\right)\right] .
\end{array}
$$

By Relation (iii) of (B.3), the terms $T_{1,1}$ and $T_{2,1}$ cancel, and $T_{2,2}$ may be written as:

$$
T_{2,2}=\sum_{K \in \mathcal{M}} \frac{|K|}{\delta t} \phi\left(z_{K}\right)\left(\rho_{K}-\rho_{K}^{*}\right)
$$

Reordering the sums in $T_{2,3}$ and using Relation (ii) of (B.3), we get:

$$
T_{2,3}=\sum_{\sigma \in \mathcal{E}_{\text {int }}, \sigma=K \mid L} F_{K, \sigma}\left[\phi\left(z_{K}\right)+\phi^{\prime}\left(z_{K}\right)\left(z_{\sigma}-z_{K}\right)-\phi\left(z_{L}\right)-\phi^{\prime}\left(z_{L}\right)\left(z_{\sigma}-z_{L}\right)\right] .
$$


Without loss of generality, let us suppose that we have chosen, in the last sum, the orientation of $\sigma=K \mid L$ in such a way that $F_{K, \sigma} \geq 0$. We thus get, since $z_{\sigma}=z_{K}$ :

$$
\begin{aligned}
\left.\delta \phi\right|_{\sigma}=\phi\left(z_{K}\right)+\phi^{\prime}\left(z_{K}\right)\left(z_{\sigma}-z_{K}\right)-\phi\left(z_{L}\right)-\phi^{\prime}\left(z_{L}\right)\left(z_{\sigma}-z_{L}\right) \\
=\phi\left(z_{K}\right)-\left[\phi\left(z_{L}\right)+\phi^{\prime}\left(z_{L}\right)\left(z_{K}-z_{L}\right)\right],
\end{aligned}
$$

which is non-negative by convexity of $\phi$. Finally, we thus get:

$$
T_{1}+T_{2} \geq \sum_{K \in \mathcal{M}} \frac{|K|}{\delta t}\left[\rho_{K}^{*}\left(\phi\left(z_{K}\right)-\phi\left(z_{K}^{*}\right)\right)+\phi\left(z_{K}\right)\left(\rho_{K}-\rho_{K}^{*}\right)\right],
$$

which concludes the proof.

Remark B.2 (Other choices for $z_{\sigma}$ ). In fact, Inequality of Proposition B.1 holds for any choice such that the quantity $\left.\delta \phi\right|_{\sigma}$ defined in its proof is non-negative, which may be written as:

$$
\left[\phi^{\prime}\left(z_{K}\right)-\phi^{\prime}\left(z_{L}\right)\right]\left(z_{\sigma}-z_{K}\right) \geq \phi\left(z_{L}\right)+\phi^{\prime}\left(z_{L}\right)\left(z_{K}-z_{L}\right)-\phi\left(z_{K}\right) .
$$

Let us suppose that $\phi$ is twice continuously differentiable. There exists $\bar{z}_{\sigma}$ and $\overline{\bar{z}}_{\sigma}$, both lying between $z_{K}$ and $z_{L}$ and such that:

$$
\begin{aligned}
& \phi^{\prime}\left(z_{K}\right)=\phi^{\prime}\left(z_{L}\right)+\phi^{\prime \prime}\left(\bar{z}_{\sigma}\right)\left(z_{K}-z_{L}\right), \\
& \phi\left(z_{K}\right)=\phi\left(z_{L}\right)+\phi^{\prime}\left(z_{L}\right)\left(z_{K}-z_{L}\right)+\frac{1}{2} \phi^{\prime \prime}\left(\overline{\bar{z}}_{\sigma}\right)\left(z_{K}-z_{L}\right)^{2} .
\end{aligned}
$$

Let us now define $\theta$ such that $z_{\sigma}-z_{K}=\theta\left(z_{L}-z_{K}\right)$. With this notations, we obtain that $\left.\delta \phi\right|_{\sigma} \geq 0$ is equivalent to:

$$
\theta \leq \frac{1}{2} \frac{\phi^{\prime \prime}\left(\overline{\bar{z}}_{\sigma}\right)}{\phi^{\prime \prime}\left(\bar{z}_{\sigma}\right)}
$$

By convexity of $\phi$, the upwind choice (i.e. $\theta=0$ ) always satisfies this relation, which is consistent with Proposition B.1. In addition, we see that, for $\phi(s)=s^{2}$, the choice $\theta=1 / 2$ is possible: in other words, as proven in [15], the centered choice ensures the kinetic energy conservation.

\section{REFERENCES}

[1] P. Bénilan, L. Boccardo, T. Gallouët, R. Gariepy, M. Pierre, and J.-L. Vazquez. An $L^{1}$-theory of existence and uniqueness of solutions of nonlinear elliptic equations. Annali della Scuola Normale Superiora di Pisa, Classe de Scienze, 22:240-273, 1995.

[2] D. Blanchard and F. Murat. Renormalized solutions of nonlinear parabolic problems with $L^{1}$ data: existence and uniqueness. Proceedings of the Royal Society of Edinburgh Section A, 127:1137-1152, 1997.

[3] L. Boccardo, A. Dall'Aglio, T. Gallouët, and L. Orsina. Nonlinear parabolic equations with measure data. Journal of Functional Analysis, 147:237-258, 1997.

[4] L. Boccardo and T. Gallouët. Non-linear elliptic and parabolic equations involving measure data. Journal of Functional Analysis, 87:149-169, 1989.

[5] F. Boyer and P. Fabrie. Eléments d'analyse pour l'étude de quelques modèles d'écoulements de fluides visqueux incompressibles. In Mathématiques et Applications. Springer, 2006.

[6] A. Bradji and R. Herbin. Discretization of the coupled heat and electrical diffusion problems by the finite element and the finite volume methods. IMA Journal of Numerical Analysis, 28:469-495, 2008.

[7] J. Casado-Díaz, T. Chacón Rebollo, V. Girault, M. Gómez Mármol, and F. Murat. Finite elements approximation of second order linear elliptic equations in divergence form with right-hand side in $L^{1}$. Numerische Mathematik, 105:337-374, 2007.

[8] S. Clain. Analyse mathématique et numérique d'un modèle de chauffage par induction. $\mathrm{PhD}$ thesis, EPFL, 1994. 
[9] Y. Coudière, T. Gallouët, and R. Herbin. Discrete Sobolev Inequalities and $L^{p}$ Error Estimates for Approximate Finite Volume Solutions of Convection Diffusion Equations. Mathematical Modelling and Numerical Analysis, 35:767-778, 1998.

[10] J. Droniou, T. Gallouët, and R. Herbin. A finite volume scheme for a noncoercive elliptic equation with measure data. SIAM Journal on Numerical Analysis, 41:1997-2031, 2003.

[11] J. Droniou and A. Prignet. Equivalence between entropy and renormalized solutions for parabolic equations with soft measure data. Nonlinear Differential Equations and Applications, 14:181-205, 2007.

[12] R. Eymard, T. Gallouët, and R. Herbin. Finite volume methods. In P. Ciarlet and J.L. Lions, editors, Handbook of Numerical Analysis, Volume VII, pages 713-1020. North Holland, 2000.

[13] R. Eymard, T. Gallouët, and R. Herbin. Discretisation of heterogeneous and anisotropic diffusion problems on general nonconforming meshes - SUSHI: a scheme using stabilization and hybrid interfaces. IMA Journal of Numerical Analysis, 30:1009-1043, 2010.

[14] R. Eymard, R. Herbin, and J.-C. Latché. Convergence analysis of a colocated finite volume scheme for the incompressible Navier-Stokes equations on general 2D or 3D meshes. SIAM Journal on Numerical Analysis, 45:1-36, 2007.

[15] T. Gallouët, L. Gastaldo, R. Herbin, and J.-C. Latché. An unconditionally stable pressure correction scheme for compressible barotropic Navier-Stokes equations. Mathematical Modelling and Numerical Analysis, 42:303-331, 2008.

[16] T. Gallouët and R. Herbin. Finite volume approximation of elliptic problems with irregular data. In Finite Volume for Complex Applications II. Hermes, 1999.

[17] T. Gallouët and R. Herbin. Convergence of linear finite elements for diffusion equations with measure data. Comptes Rendus de l'Académie des Sciences de Paris, Série I, Mathématiques, 338:81-84, 2004.

[18] L. Gastaldo, R. Herbin, and J.-C. Latché. A discretization of phase mass balance in fractional step algorithms for the drift-flux model. IMA Journal of Numerical Analysis, 31:116-146, 2011.

[19] J.-L. Lions. Quelques méthodes de résolution des problèmes aux limites non linéaires. Dunod, Paris, 1969.

[20] A. Prignet. Existence and uniqueness of "entropy" solutions of parabolic problems with $L^{1}$ data. Nonlinear Analysis, 28:1943-1954, 1997.

Université De Provence

E-mail address: gallouet@cmi.univ-mrs.fr

Institut de RAdioprotection et de SÛReté Nucléaire (IRSN)

E-mail address: aurelien.larcher@irsn.fr

Institut de RAdioprotection et DE SÛRETÉ NuclÉAire (IRSN)

E-mail address: jean-claude.latche@irsn.fr 